\title{
Strong inhibitory signaling underlies stable temporal dynamics and working memory in spiking neural networks
}

\author{
Robert Kim ${ }^{\mathbf{1 , 2 , 3}}$ *and Terrence J. Sejnowski ${ }^{\mathbf{1 , 4 , 5}}$ *
}

${ }^{1}$ Computational Neurobiology Laboratory, Salk Institute for Biological Studies, La Jolla, CA 92037, USA

2 Neurosciences Graduate Program, University of California San Diego, La Jolla, CA 92093, USA

3 Medical Scientist Training Program, University of California San Diego, La Jolla, CA 92093, USA

${ }^{4}$ Institute for Neural Computation, University of California San Diego, La Jolla, CA 92093, USA

${ }^{5}$ Division of Biological Sciences, University of California San Diego, La Jolla, CA 92093, USA

* Correspondence: rkim@salk.edu (R.K.), terry@salk.edu (T.J.S.)

\begin{abstract}
Cortical neurons process information on multiple timescales, and areas important for working memory (WM) contain neurons capable of integrating information over a long timescale. However, the underlying mechanisms for the emergence of neuronal timescales stable enough to support WM are unclear. By analyzing a spiking recurrent neural network (RNN) model trained on a WM task and activity of single neurons in the primate prefrontal cortex, we show that the temporal properties of our model and the neural data are remarkably similar. Dissecting our RNN model revealed strong inhibitory-to-inhibitory connections underlying a disinhibitory microcircuit as a critical component for long neuronal timescales and WM maintenance. We also found that enhancing inhibitory-to-inhibitory connections led to more stable temporal dynamics and improved task performance. Finally, we show that a network with such microcircuitry can perform other tasks without disrupting its pre-existing timescale architecture, suggesting that strong inhibitory signaling underlies a flexible WM network.
\end{abstract}




\section{Introduction}

2 Temporal receptive fields are hierarchically organized across the cortex [1, 2]. Areas important for

3 higher cognitive functions are capable of integrating and processing information in a robust manner

4 and reside at the top of the hierarchy $[1-3]$. The prefrontal cortex (PFC) is a higher-order cortical

5 region that supports a wide range of complex cognitive processes including working memory (WM),

6 an ability to encode and maintain information over a short period of time 44, 5]. However, the

7 underlying circuit mechanisms that give rise to stable temporal receptive fields strongly associated

8 with WM are not known and experimentally challenging to probe. A better understanding of

9 possible mechanisms could elucidate not only how areal specialization in the cortex emerges but

10 also how local cortical microcircuits carry out WM computations.

11 Previous experimental studies reported that baseline activities of single neurons in the primate

12 PFC contained unique temporal receptive field structures. Using decay time constants of spike-

13 count autocorrelation functions obtained from neurons at rest, these studies demonstrated that

14 the primate PFC is mainly composed of neurons with large time constants or timescales [1, 6 8 ].

15 In addition, neurons with longer timescales carried more information during the delay period of

16 a WM task compared to short timescale neurons [8]. Chaudhuri et al. [2] proposed a large-scale

17 computational model where heterogeneous timescales were naturally organized in a hierarchical

18 manner that closely matched the hierarchy observed in the primate neocortex. The framework

19 utilized a gradient of recurrent excitation to establish varying degrees of temporal dynamics [2].

20 Although their findings suggest that recurrent excitation is correlated with area-specific timescales,

21 it is still unclear if recurrent excitation indeed directly regulates neuronal timescales and WM

22 computations.

23 Recent experimental studies paint a different picture where diverse inhibitory interneurons

24 form intricate microcircuits in the PFC to execute memory formation and retrieval 9 - 13]. Both

25 somatostatin (SST) and vasoactive intestinal peptide (VIP) interneurons have been shown to form

26 a microcircuit that can disinhibit excitatory cells via inhibition of parvalbumin (PV) interneurons

27 14, 15. Furthermore, SST and VIP neurons at the center of such disinhibitory microcircuitry were

28 causally implicated with impaired associative and working memory via optogenetic manipulations

29 9, 10, 12, 13. Consistent with these observations, the primate anterior cingulate cortex, which is 
at the top of the timescale hierarchy [1], was found to contain more diverse and stronger inhibitory inputs compared to the lateral PFC [16]. A recent theoretical study also showed that inhibitoryto-inhibitory synapses, although much fewer in number compared to excitatory connections, is a critical component for implementing robust maintenance of memory patterns 17 .

In order to characterize how strong inhibitory signaling enables WM maintenance and leads to slow temporal dynamics, we constructed a spiking recurrent neural network (RNN) model to perform a WM task and compared the emerging timescales with the timescales derived from the prefrontal cortex of rhesus monkeys trained to perform similar WM tasks. Here, we show that both primate PFC and our RNN model utilize units with long timescales to sustain stimulus information. By analyzing and dissecting the RNN model, we illustrate that inhibitory-to-inhibitory synapses incorporated into a disinhibitory microcircuit tightly control both neuronal timescales and WM task performance. Finally, we show that the primate PFC exhibits signs that it is already equipped with strong inhibitory connectivity even before learning the WM task, implying that a gradient of recurrent inhibition could naturally result in functional specialization in the cortex. We confirm this with our model and show that the task performance of RNNs with short timescales can be enhanced via increased recurrent inhibitory signals. Overall, our work offers timely insight into the role of diverse inhibitory signaling in WM and provides a circuit mechanism that can explain previously observed experimental findings.

\section{Results}

Spiking recurrent neural network model. To study how stable temporal dynamics associated with WM emerge, we trained a spiking RNN model to perform a WM task. The model used in the present study is composed of leaky integrate-and-fire (LIF) units recurrently connected to one another (see Methods).

The WM task we used to train the spiking RNNs was a delayed match-to-sample (DMS) task (Fig. 1 ; see Methods). The task began with a $1 \mathrm{~s}$ long fixation period (i.e., no external input)

followed by two sequential input stimuli (each stimulus lasting for $0.25 \mathrm{~s}$ ) separated by a delay period $(0.75 \mathrm{~s})$. The input signal was set to either -1 or +1 during the stimulus window. If the 57 two sequential stimuli had the same sign $(-1 /-1$ or $+1 /+1)$, the network was trained to produce an 8 output signal approaching +1 after the offset of the second stimulus. If the stimuli had opposite 


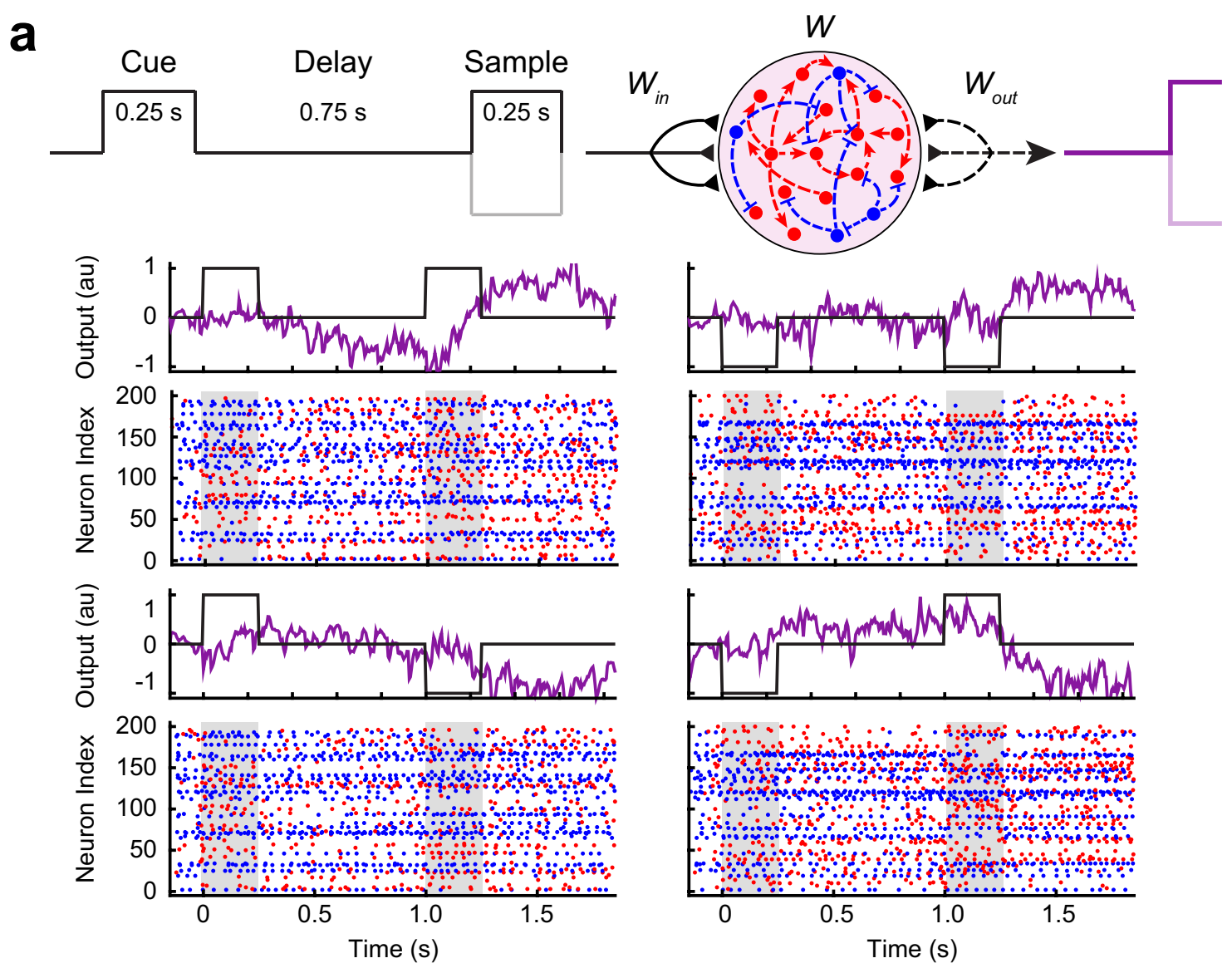

b

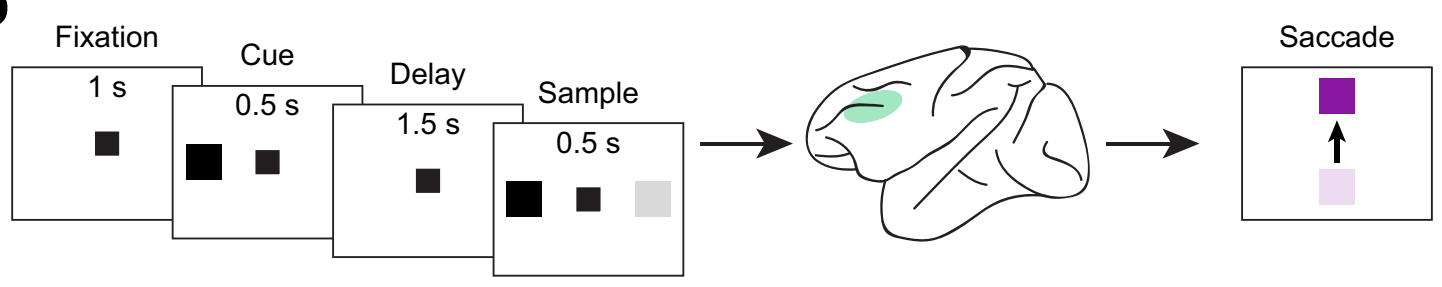

Fig. 1 | Recurrent neural network model and experimental data. a, Spiking recurrent neural network (RNN) model contained excitatory (red circles) and inhibitory (blue circles) units recurrently connected to one another. The model was trained to perform a delayed match-to-sample (DMS) task. Each RNN contained 200 units (80\% excitatory and $20 \%$ inhibitory), and 40 RNNs were trained to perform the DMS task. The dashed lines (recurrent connections and readout weights) were optimized via a supervised learning method. Example output signals along with the corresponding spike raster plots shown. Gray shading, stimulus window. b, Spatial DMS task paradigm used by Constantinidis et al. [18 to train four rhesus monkeys. Extracellular recordings from the dorsolateral prefrontal cortex (green area) were analyzed.

59 signs $(-1 /+1$ or $+1 /-1)$, the network produced an output signal approaching -1 .

60 Using a method that we had previously developed, we configured the recurrent connections

61 required for the spiking model to perform the task 19. Briefly, we trained continuous-variable rate

62 RNNs to perform the task using a gradient descent algorithm, and the trained networks were then 
a

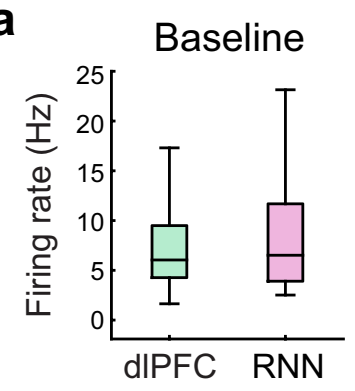

C

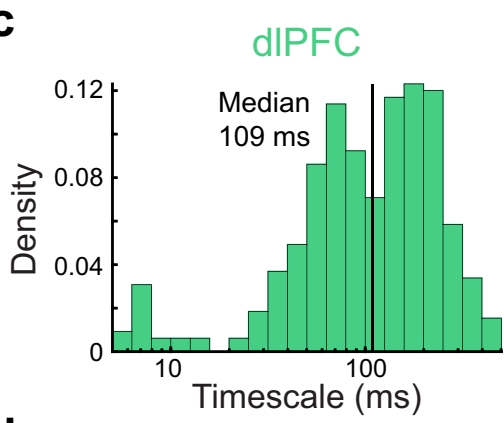

d

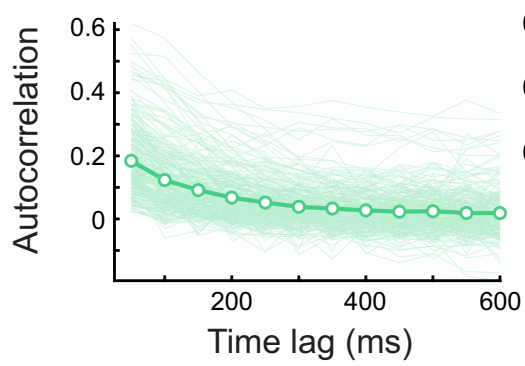

Short $\tau$

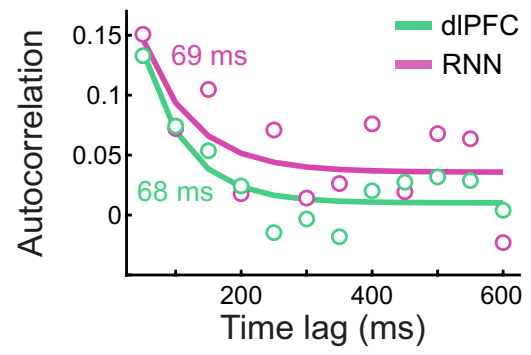

Trained RNNs
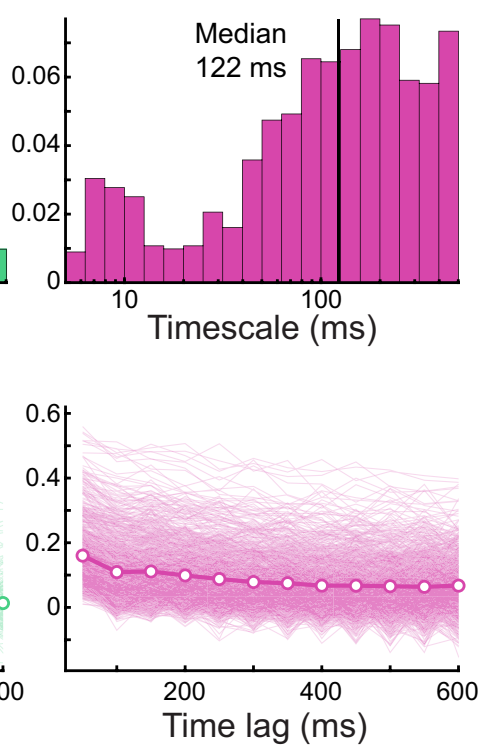

Long $\tau$

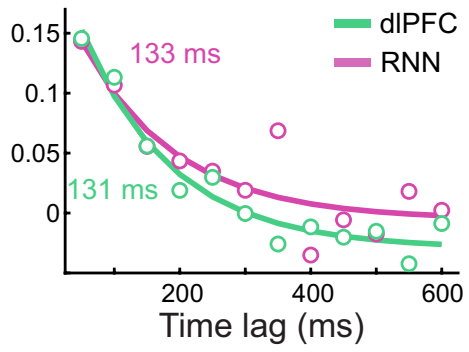

Untrained RNNs
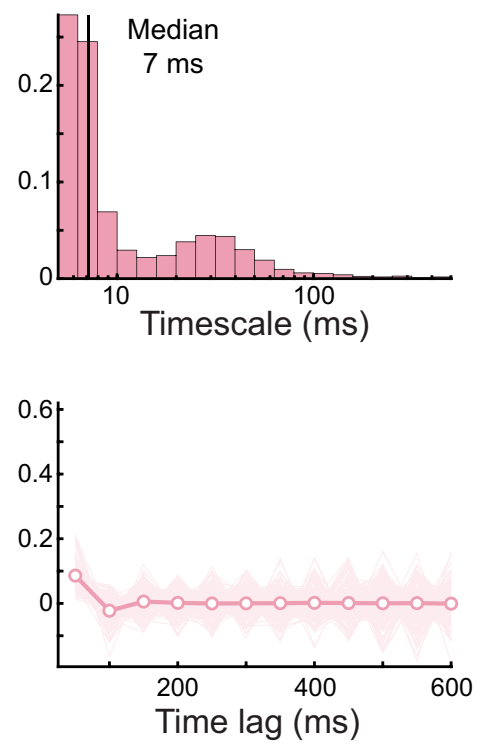

Fig. 2 | RNN model trained on the DMS task and the dlPFC data contain units with long timescales. a, Distribution of the firing rates during the fixation period was not significantly different between the experimental data and the RNN model $(P<0.70$, two-sided Wilcoxon rank-sum test). b, Autocorrelation decay curves from example units with short (left) and long (right) timescale values. c, Histograms of the distribution of the timescales from the experimental data $(n=325$; green), trained RNNs ( $n=931$; magenta), and random RNNs $(n=3963$; light magenta). Solid vertical lines represent median $\log (\tau)$. d, Autocorrelation decay curves from single units (light) and the population average autocorrelation (bold) for the dlPFC data, trained RNNs, and random RNNs. For the random RNNs, only $20 \%$ of the total single unit traces shown. Boxplot central lines, median; red circles, mean; bottom and top edges, lower and upper quartiles; whiskers, $1.5^{*}$ interquartile range; outliers not plotted.

mapped to LIF networks. In total, we "trained" 40 LIF RNNs of 200 units (80\% excitatory and $20 \%$ inhibitory units) to perform the task with high accuracy (accuracy $>95 \%$; see Methods).

Experimental data. To ensure that our spiking model is a biologically valid one for probing neuronal timescales observed in the cortex, we also analyzed a publicly available dataset containing extracellular spike trains recorded from the dorsolateral prefrontal cortex (dlPFC) of four rhesus monkeys $18,20,21$. The monkeys were trained on spatial and feature DMS tasks. A trial for both 
a

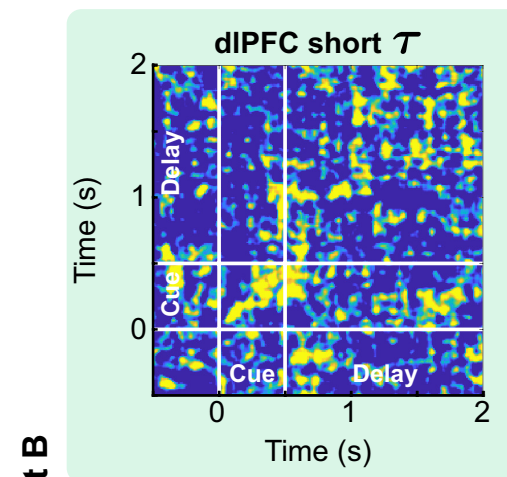

$\stackrel{m}{\frac{m}{0}}$

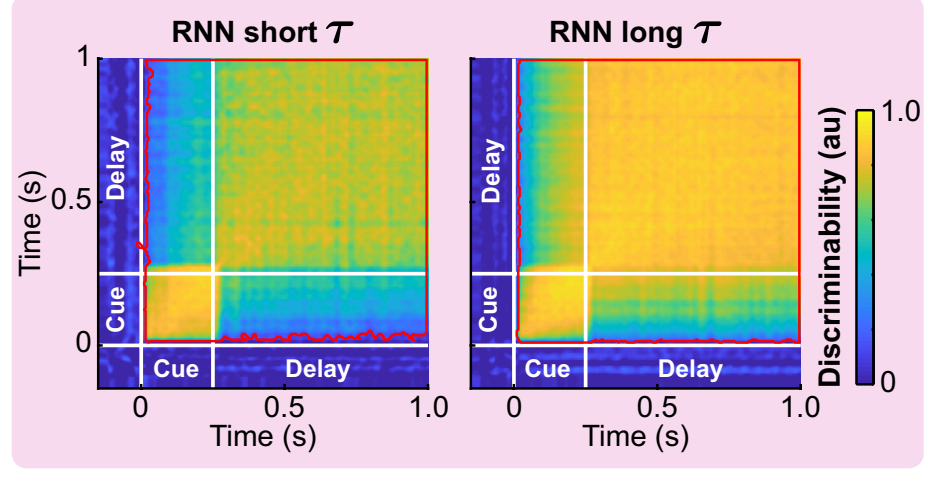

b
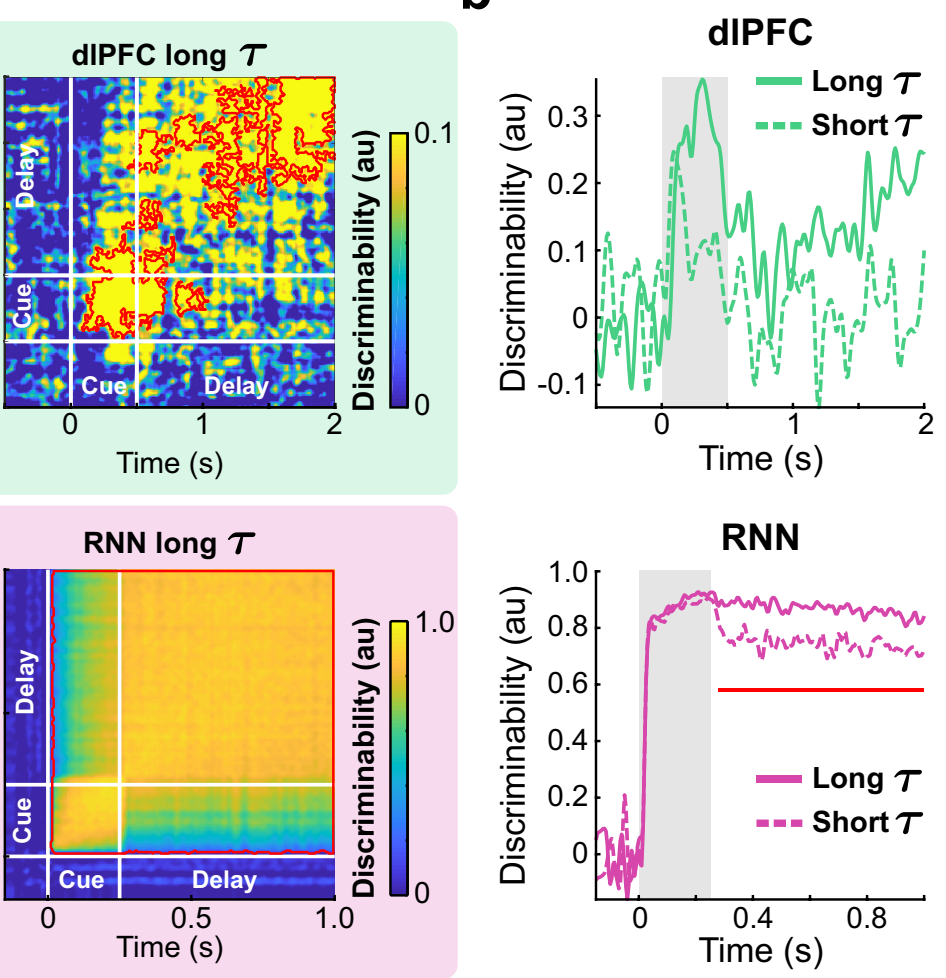

\section{Split A}

Fig. 3 | Long $\tau$ units maintain cue stimulus information during the delay period robustly. a, Cross-temporal discriminability matrices for the dlPFC data (top row) and the RNN model (bottom row). Red contours indicate significant decodability (cluster-based permutation test, $P<0.05$; see Methods). $\mathbf{b}$, Within-time discriminability timecourses from the short (dashed) and long (solid) $\tau$ groups for the dlPFC data and the RNN model. Gray shading, cue stimulus window. Red lines indicate significant differences in decoding between the short and long $\tau$ groups (cluster-based permutation test, $P<0.05$; see Methods).

69 task types began with a fixation period ( $1 \mathrm{~s}$ in duration) during which the monkeys were required

70 to maintain their gaze at a fixation target. For a spatial DMS trial, the monkeys were trained

71 to report if two sequential stimuli separated by a delay period $(1.5 \mathrm{~s})$ matched in spatial location

72 (Fig. 1b). For a feature DMS trial, the monkeys were required to distinguish if two sequential

73 stimuli (in the same spatial location) matched in shape. More details regarding the dataset and

74 the tasks can be found in the Methods and in Qi et al. 20] and Meyer et al. 21.

75 Long neuronal timescales in both RNN model and experimental data. Using the spike-

76 count autocorrelation decay time constant as a measure of a neuron's timescale, previous studies

77 demonstrated that higher cortical areas consist of neurons with long, heterogeneous timescales

78 [1, 7, 8]. Here, we sought to confirm that our spiking RNNs trained on the DMS task and the

79 neural data were also composed of units with predominantly long timescales. For each unit from 
our RNNs and the dlPFC, we computed the autocorrelation decay time constant $(\tau)$ of its spikecount during the $1 \mathrm{~s}$ fixation period (see Methods) [1]. The baseline activities (average firing rates during the fixation period) of the units that satisfied the inclusion criteria were comparable between the dlPFC data and our model (Fig. 2a; see Methods). Both data contained units with slow temporal dynamics (i.e., long $\tau$ values) and short $\tau$ units whose autocorrelation function decayed fast (Fig. 2p). Furthermore, the distribution of the timescales was heavily left-skewed for both data (Fig. 2 ,d, left and middle panels) underscoring overall slow temporal properties associated with WM. On the other hand, random RNNs (sparse, random Gaussian connectivity weights) were dominated by units with extremely short timescales (Fig. 2, d, right panels), suggesting that the long $\tau$ units observed in the trained RNNs were the result of the supervised training.

Long neuronal timescales are essential for stable coding of stimuli. Next, we investigated to see if units with longer $\tau$ values were involved with more stable coding compared to short $\tau$ units using cross-temporal decoding analysis $[8,22,23$. Briefly, for each cue stimulus identity, the trials of each unit were divided into two splits in an interleaved manner (i.e., even vs. odd trials). All possible pairwise differences (in instantaneous firing rates) between cue conditions were computed within each split. Finally, a Fisher-transformed Pearson correlation coefficient was computed between the pairwise differences of the first split at time $t_{1}$ and the differences of the second split at time $t_{2}$ (see Methods). Therefore, a high Fisher-transformed correlation value (i.e., high discriminability) represents a reliable cue-specific difference present in the network population.

We performed the above analysis on short and long neuronal timescale subgroups from the neural data and the RNN model. A unit was assigned to the short $\tau$ group if its timescale was smaller than the lower quartile value. The upper quartile was used to identify units with large $\tau$ values. There were 64 units in each subgroup for the experimental data. For the RNN model, there were 230 units in each subgroup.

The cross-temporal discriminability analysis revealed that stronger cue-specific differences (i.e., higher discriminability) across the delay period were present in the long $\tau$ subgroup compared to the short $\tau$ subgroup for both data $($ Fig. 3a). The significant decodability during the delay period for the dlPFC dataset mainly stemmed from the spatial task dataset Supplementary Fig. 1. The within-time discriminability (i.e., taking the diagonal values of the cross-temporal decoding 


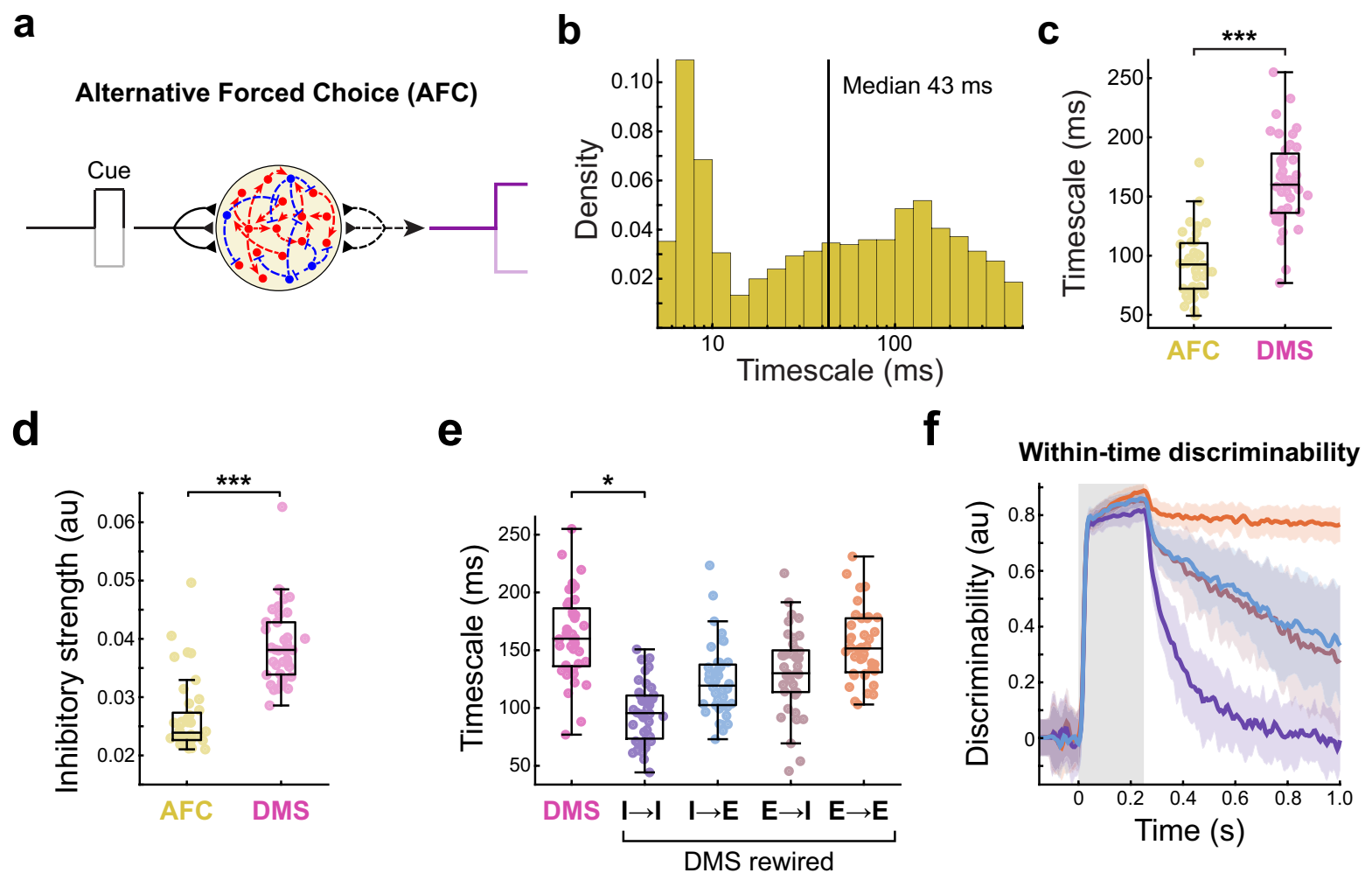

Fig. 4 | Inhibitory synaptic weights lead to task-specific timescales. a, Task paradigm for the alternative forced choice (AFC) task. b, Distribution of the neuronal timescales extracted from 40 RNNs trained on the AFC task. Solid vertical line represents median $\log (\tau)$. c, Average timescale values from the AFC and DMS RNNs. Each circle represents the average value from one RNN. d, Average recurrent inhibitory synaptic strengths from the AFC and DMS models. e, Average timescales from the DMS RNNs with each synaptic type rewired randomly (Friedman test, $F=74.19, P<0.0001$ ). f, Within-time discriminability timecourses averaged across all the DMS RNNs for each rewiring condition. Same color scheme as e. The bold line indicates the mean timecourse averaged across 40 RNNs (and all units). Colored shading, \pm standard deviation (s.d.). Gray shading, cue stimulus window. Boxplot central lines, median; red circles, mean; bottom and top edges, lower and upper quartiles; whiskers, $1.5 *$ interquartile range; outliers not plotted. ${ }^{*} P<0.01,{ }^{* * *} P<0.0001$ by Wilcoxon signed-rank test $(\mathbf{c}, \mathbf{d})$ or Dunn's multiple comparisons test (e).

matrices) for the long $\tau$ group was significantly higher than the discriminability observed from the

110 short $\tau$ group throughout the delay period for the RNN model (Fig. 3b). Although significant

111 within-time discriminability was not observed for the dlPFC data (Fig. 3b, top), Wasmuht et al.

112 8 reported significant within-time decodability during the delay period in the primate lateral

113 prefrontal cortex, consistent with our model findings.

114 Strong inhibitory connections give rise to task-specific temporal receptive fields. Neu-

115 ronal timescales extracted from cortical areas have been shown to closely track the anatomical

116 and functional organization of the primate cortex [1, 2]. For instance, sensory areas important for 117 detecting incoming stimuli house neurons with short timescales. On the other hand, higher cortical 

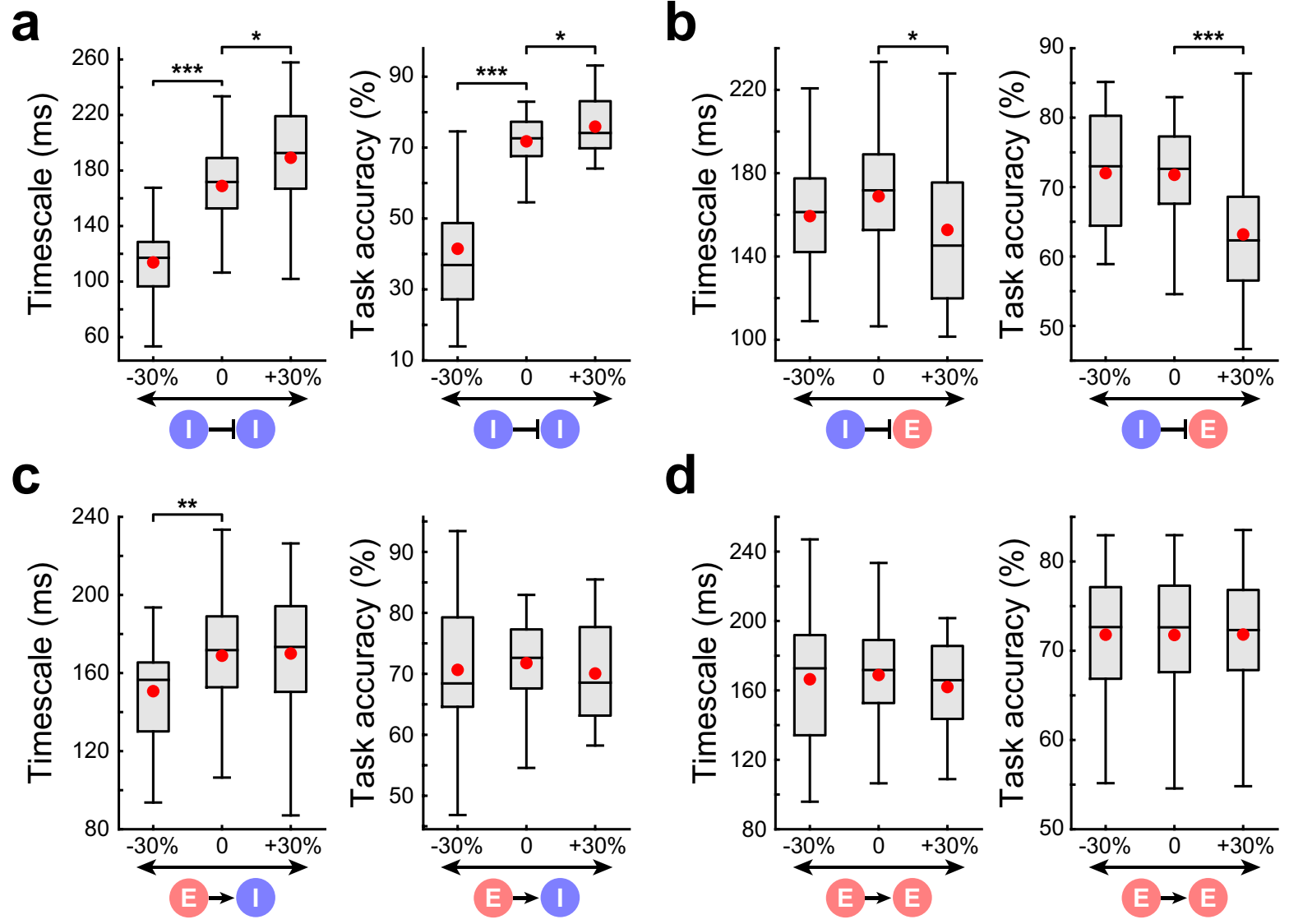

d
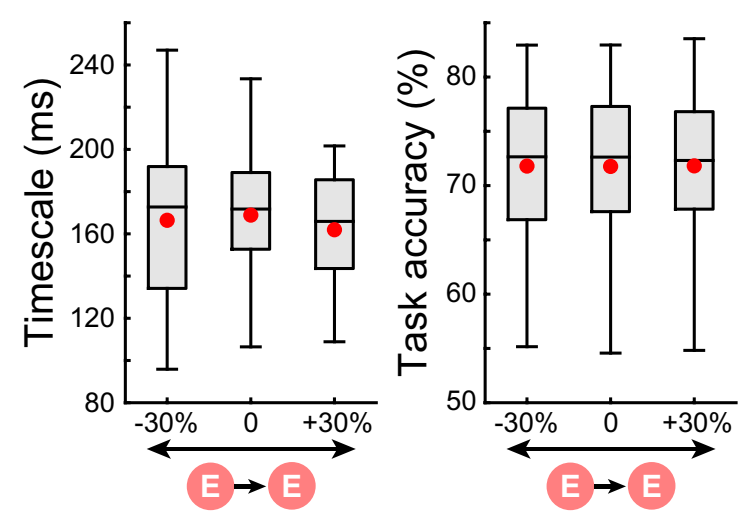

Fig. 5 $\mid I \rightarrow I$ connectivity strength strongly mediates both neuronal timescales and task performance. a, b, c, d, Timescales and task performance changes when $I \rightarrow I(\mathbf{a}), I \rightarrow E(\mathbf{b}), E \rightarrow I$ $(\mathbf{c})$, or $E \rightarrow E$ (d) connection strength was either decreased or increased by $30 \%$. Boxplot central lines, median; red circles, mean; bottom and top edges, lower and upper quartiles; whiskers, 1.5 *interquartile range; outliers not plotted. ${ }^{*} P<0.05,{ }^{* *} P<0.005,{ }^{* * *} P<0.0001$ by Wilcoxon signed-rank test.

118 areas, including prefrontal areas, may require neurons with stable temporal receptive fields that

119 are capable of encoding and integrating information over a longer timescale.

120 To investigate if such functional specialization also emerges in our spiking model, we trained

121 another group of spiking RNNs ( $n=40 \mathrm{RNNs}$ ) on a simpler task that did not require WM. The

122 non-WM task, which we refer to as two-alternative forced choice (AFC) task, required the RNNs to

123 respond immediately after the cue stimulus: output approaching -1 for the "- 1 " cue and +1 for the

124 "+1" cue (Fig. 4 a; see Methods). Apart from the task paradigm, all the other model parameters

125 were identical to the parameters used for the DMS RNNs.

126 Because the AFC task paradigm did not require the RNNs to store information related to the 127 cue stimulus, we expected that these networks would operate on a much faster timescale compared 
128 to the DMS RNNs. Consistent with this hypothesis, the AFC RNNs did not contain as many long

$129 \tau$ units as the DMS RNNs (Fig. 4b), and the timescales averaged by network were also significantly

130 faster for the AFC RNNs (Fig. 4r).

131 To gain insight into the circuit mechanisms underlying the difference in the timescale distribu-

132 tions of the AFC and DMS RNN models, we compared the recurrent connectivity patterns between

133 these two models. Among other differences, the most notable difference was the inhibitory synaptic

134 strength, which was significantly greater for the DMS RNNs (Fig. 4 $\mathrm{d})$. In order to confirm if strong

135 inhibitory signaling led to the long timescales observed in the DMS model, we randomly rewired all

136 the connections belonging to each of the four synaptic types $(I \rightarrow I, I \rightarrow E, E \rightarrow I$, and $E \rightarrow E)$

137 and computed the timescales again (see Methods). Of the four conditions, only rewiring $I \rightarrow I$

138 synapses resulted in significantly shorter timescales than the timescales from the intact DMS model

139 (Fig. 4 ), and the distribution of the timescales pooled from all 40 RNNs with $I \rightarrow I$ connections

140 shuffled resembled the distribution obtained from the AFC model (Supplementary Fig. 2). In ad-

141 dition, the amount of cue-specific information maintained during the delay period (as measured by

142 the within-time decoding timecourses) was the lowest for the $I \rightarrow I$ rewired condition (Fig. 4 f $)$,

143 suggesting that shuffling $I \rightarrow I$ synapses was detrimental to memory maintenance.

\section{Inhibitory-to-inhibitory connections regulate both neuronal timescales and task per-}

145 formance. Given our findings that $I \rightarrow I$ connections are important for long neuronal timescales

146 and information encoding, we next investigated if $I \rightarrow I$ synapses could be manipulated to provide

147 more stable temporal receptive fields and to improve WM maintenance.

148 Recent studies revealed that optogenetically stimulating SST or VIP interneurons that specifi-

149 cally inhibit PV interneurons could improve memory retrieval 10 12. Based on these experimental

150 observations, we expected that strengthening $I \rightarrow I$ synapses would increase neuronal timescales

151 and task performance of the DMS RNNs. To test this hypothesis, we first generated another group

152 of RNNs with poor DMS task performance (26 RNNs; mean accuracy \pm s.e.m., $71.77 \pm 1.43 \%$ ).

153 Next, we modeled the effects of optogenetic manipulation of VIP/SST neurons by either decreasing

154 or increasing $I \rightarrow I$ synaptic strength $\left(W_{I \rightarrow I}\right)$ in each network by $30 \%$ (see Methods). Decreasing

155 the connection strength led to significantly shorter timescales compared to the RNNs without any

156 modification (Fig. 5a, left). Strengthening $W_{I \rightarrow I}$ resulted in a moderate but significant increase in 

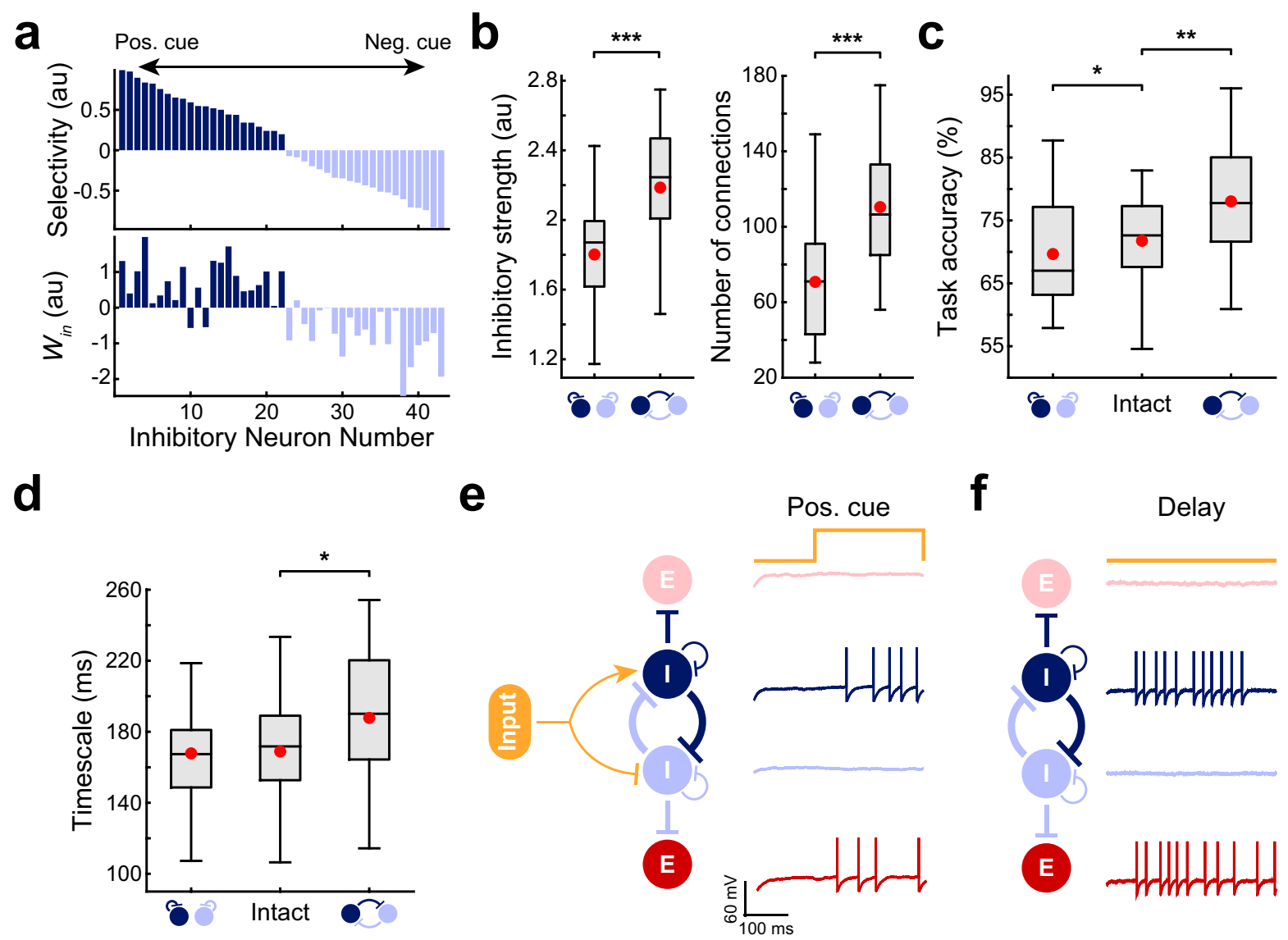

Fig. 6 | Two oppositely tuned inhibitory subgroups mutually inhibit each other for WM maintenance. a, Cue preference selectivity (top) and input weights ( $W_{i n}$; bottom) from inhibitory units of an example DMS RNN. The selectivity index values are sorted in descending order. b, Average inhibitory strengths (left) and number of inhibitory connections (right) within and across two oppositely tuned inhibitory subgroups from all 40 DMS RNNs. c, Average task performance of the DMS RNN model when the within-group or across-group inhibition was increased by $30 \%$. d, Average neuronal timescales of the DMS RNNs when the within-group or across-group inhibition was increased by $30 \%$. e, f, Schematic illustration of the circuit mechanism employed by the DMS RNN model during the cue stimulus window (e) and delay period (f). The positive cue stimulus was used as an example, and membrane voltage tracings from example units are shown. Dark blue and dark red units indicate units that prefer the positive cue stimulus, while the light blue and light red units favor the negative cue. For simplicity, only recurrent inhibitory connections are shown. Boxplot central lines, median; red circles, mean; bottom and top edges, lower and upper quartiles; whiskers, $1.5 *$ interquartile range; outliers not plotted. ${ }^{*} P<0.05$, ${ }^{* *} P<0.005,{ }^{* * *} P<0.0001$ by two-sided Wilcoxon rank-sum test $(\mathbf{b})$ or Wilcoxon signed-rank test $(\mathbf{c}, \mathbf{d})$.

157 neuronal timescale (Fig. 5a, left). The task performance of the RNNs followed the same pattern:

158 decreasing $W_{I \rightarrow I}$ severely impaired WM maintenance while increasing $W_{I \rightarrow I}$ significantly improved

159 task performance (Fig. 5a, right). Increasing $W_{I \rightarrow I}$ further did not correspond to a significant in-

160 crease in timescale and task performance (Supplementary Fig. 3). For $I \rightarrow E$ connections, only

161 enhancing $W_{I \rightarrow E}$ resulted in significant changes in both timescale and task performance (Fig. 5 b).

162 Manipulating $E \rightarrow I$ synapses did not affect the task performance, but decreasing $W_{E \rightarrow I}$ signif- 
163 icantly shortened the timescales (Fig. 5). Altering the excitatory-to-excitatory connections did

164 not produce any significant changes (Fig. 5d). Overall, these findings suggest that $I \rightarrow I$ synapses

165 tightly mediate both temporal stability and WM maintenance. The findings also indicate that the

166 main downstream effect of $I \rightarrow I$ connections is to disinhibit excitatory units.

167 Unique inhibitory-to-inhibitory circuitry for WM maintenance. So far, our results indicate

168 that (1) microcircuitry involving specific $I \rightarrow I$ connectivity patterns is important for WM (Fig. 4)

169 and (2) $I \rightarrow I$ can be strengthened to enhance both neuronal timescales and task performance

170 (Fig. 5a). Here, we dissect the DMS RNN model to elucidate how specific and strong $I \rightarrow I$

171 connections lead to stable memory retention.

172 Focusing on inhibitory units only, we first characterized the cue stimulus selectivity from each

173 inhibitory unit in an example DMS network (see Methods). Analyzing the selectivity index values

174 revealed two distinct subgroups of inhibitory units in the network: one group of units favoring the

175 positive cue stimulus and the other group selective for the negative stimulus (Fig. 6a, top). The

176 input weights $\left(W_{i n}\right)$ that project to these units closely followed the selectivity pattern $[$ Fig. 6 ,

177 bottom).

178 Given these two subgroups with distinct selectivity patterns, we next hypothesized that mutual

179 inhibition between these two groups (across-group inhibition) was stronger than within-group in-

180 hibition. Indeed, inhibition between the oppositely tuned inhibitory populations was significantly

181 greater (both in synaptic strength and number of connections) than inhibition within each sub-

182 group across all RNNs (Fig. 6b). To confirm that the behavioral improvement we observed with

$183 I \rightarrow I$ enhancement in Fig. 5a was largely due to the strengthened across-group inhibition, we

184 increased across-group and within-group $I \rightarrow I$ connections separately (see Methods). The DMS

185 RNN performance improved following enhancement of the across-group inhibition, while increas-

186 ing the within-group inhibition impaired performance (Fig. 6). In addition, across-group $I \rightarrow I$

187 enhancement resulted in a significant increase in neuronal timescale (Fig. 6d).

188 In summary, these findings imply that robust inhibition of oppositely tuned inhibitory sub-

189 populations is critical for memory maintenance in our RNN model. For example, a positive cue

190 stimulus activates the inhibitory subgroup selective for that stimulus and deactivates the negative

191 stimulus subgroup (Fig. 6e). Through disinhibition, a group of excitatory units that favor the 
a

Low variability High variability
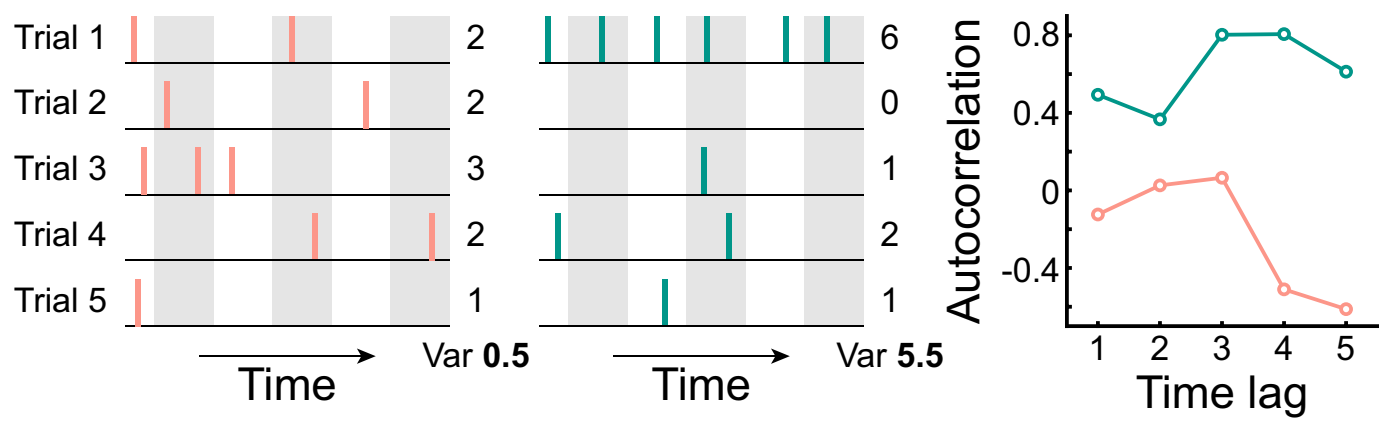

b

dIPFC

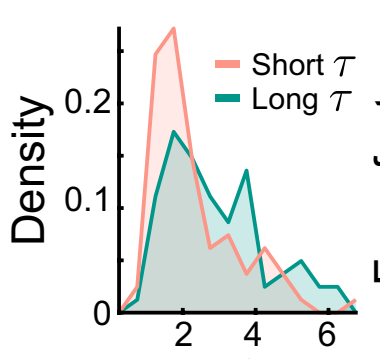

Fano factor

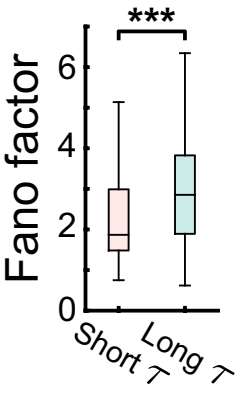

RNN
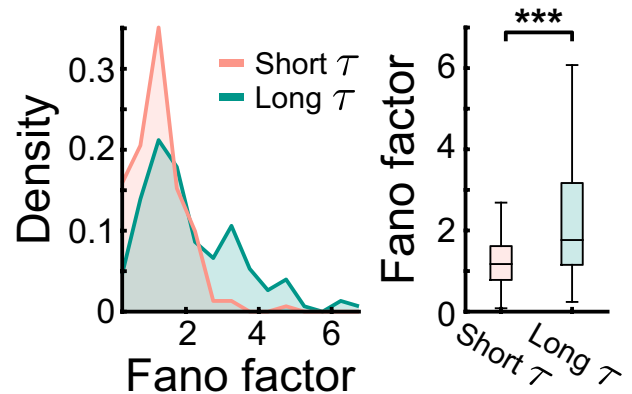

C

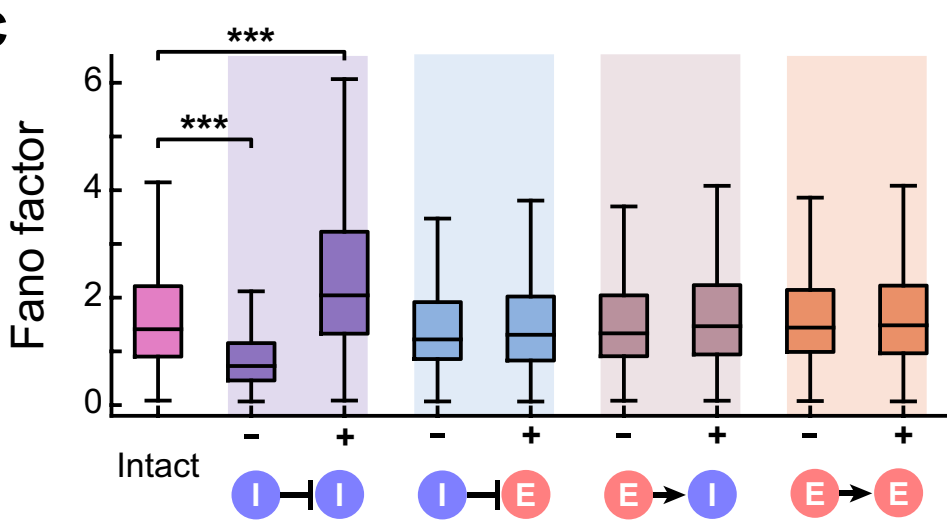

d

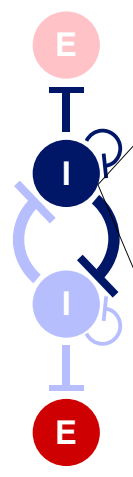

Fixation

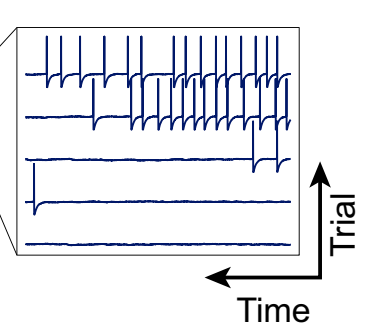

(1)

Fig. 7 | High trial-to-trial spike-count variability during the fixation corresponds to long neuronal timescale. a, Schematic illustrating how high spike-count variability across multiple trials can result in a slow decay of the autocorrelation function. $\mathbf{b}$, Comparison of the spike-count Fano factors from the short and long $\tau$ groups in the neural data (left) and the DMS RNN model (right). c, Average Fano factors from the DMS model with each of the synaptic type either decreased ("-") or increased ("+") by $30 \%$ (Kruskal-Wallis test, $H=665.2, P<0.0001$ ). d, Spiking activity of an example inhibitory unit during the fixation period across 5 trials. The trials were sorted by the number of spikes. Units that were strongly modulated by the disinhibitory circuit mechanism showed highly dynamic baseline firing patterns across trials. Boxplot central lines, median; red circles, mean; bottom and top edges, lower and upper quartiles; whiskers, $1.5 *$ interquartile range; outliers not plotted. ${ }^{* * *} P<0.0001$ by two-sided Wilcoxon rank-sum test (b) or Dunn's multiple comparisons test (c). 
192 positive cue stimulus also emerges. During the delay period, the inhibition strength between these

193 two inhibitory subgroups dictates the stability of the cue-specific activity patterns generated during

194 the stimulus window (Fig. 6f).

195 Circuit mechanism for WM generates units with long neuronal timescales. The circuit

196 mechanism (Fig. 6e,f) explains why enhancing $I \rightarrow I$ connections results in improved WM perfor-

197 mance, but it is still not clear how this same mechanism also produces units with long timescales.

198 Here, we first demonstrate that a high trial-to-trial spike-count variability during the fixation

199 period could give rise to slow decay of the spike-count autocorrelation function. If a neuron exhibits

200 highly variable activity patterns across many trials such that it is highly active (i.e., persistent

201 firing) in some trials and relatively silent in other trials, the Pearson correlation between any two

202 time bins within the fixation window could be large (Fig. 7 a). On the other hand, firing activities

203 with a low trial-to-trial variability could result in a weak correlation between two time bins. To

204 directly test this positive relationship between trial-to-trial variability and neuronal timescales, we

205 computed spike-count Fano factors (spike-count variance divided by spike-count mean across trials;

206 see Methods) for the short and long $\tau$ subgroups in both neural and model data. The Fano factor

207 values for the short timescale subgroup were significantly smaller than the values obtained from

208 the long $\tau$ group for both data (Fig. $7 \mathrm{~b})$. There was also a significant positive correlation between

209 the spike-count Fano factors and neuronal timescales across all the units in both data (Spearman

210 rank correlation, $r=0.25, P<0.0001$ for dlPFC; $r=0.28, P<0.0001$ for RNN; Supplementary

211 Fig. 4).

212 Manipulating each of the four synaptic types (decreasing or increasing synaptic strength by

$21330 \%$ ) in our DMS RNN model revealed that $I \rightarrow I$ connections strongly modulated the spike-count

214 Fano factors (Fig. 7c). Enhancing $I \rightarrow I$ synaptic strength led to units with more variable spiking

215 patterns across trials, whereas reducing the strength resulted in smaller Fano factors (example

216 shown in Supplementary Fig. 5.

217 In our RNN model, strong $I \rightarrow I$ synapses could give rise to both excitatory and inhibitory

218 units behaving in a highly variable manner during the fixation period (Fig. 7d). For instance,

219 an inhibitory unit selective for the positive stimulus could be partially activated in some trials

220 by chance (i.e., via random noise during the fixation period), and this, in turn, could silence a 
221 portion of the negative stimulus inhibitory population (light blue circle in Fig. $7 \mathrm{~d}$ ). This leads to

222 variable firing activities across trials in inhibitory units. Furthermore, the dynamic activity of the

223 inhibitory population could be transferred to the excitatory population via disinhibition. Therefore,

$224 I \rightarrow I$ connections play a central role in conferring the network with highly dynamic baseline firing

225 patterns, which then translate to high $\tau$ values.

226 Strong $I \rightarrow I$ is an intrinsic property of prefrontal cortex. Finally, we wanted to investigate

227 whether microcircuitry with strong inhibitory-to-inhibitory synapses emerges via learning-related

228 plastic changes. Because extensive plastic changes could disrupt neurons with stable temporal

229 receptive fields, we reasoned that prefrontal cortical areas and other higher cognitive areas are en-

230 dowed with strong $I \rightarrow I$ connections whose connectivity patterns do not undergo significant plastic

231 changes during learning. Instead, learning-related changes occur to the connections stemming from

232 upstream networks that project to these areas. In order to test this hypothesis, we extracted neu-

233 ronal timescales from the recordings obtained from the same monkeys before they were trained to

234 perform the DMS tasks. In this passive paradigm (Fig. 8a), the monkeys were trained to maintain

235 their gaze at a central fixation point throughout the trial regardless of the stimuli presented around

236 the fixation point 24 .

237 Surprisingly, the timescales from the spike-train data from the dlPFC of the same four monkeys

238 that learned the passive task were similar to the timescales obtained after the monkeys learned the

239 DMS task (Fig. 8b). In addition, the cue-specific information maintenance during the delay period

240 by long $\tau$ units was largely abolished, and the within-time decoding was similar between long $\tau$ and

241 short $\tau$ neurons (Fig. 8). These findings suggest that the primate dlPFC was already equipped

242 with stable temporal receptive fields and that learning the DMS task resulted in long $\tau$ neurons

243 carrying more information during the delay period while preserving the network temporal dynamic

244 architecture.

245 Next, we asked if we could only optimize the upstream connections (i.e., input weights; $W_{\text {in }}$ ) of

246 the DMS RNNs to perform a passive version of the DMS task (Fig. 8d; see Methods). By freezing

247 the recurrent connections $(W)$, we ensured the previously observed distribution of the timescales

248 (Fig. 2b) was preserved. Re-tuning the recurrent connections for the passive task resulted in

249 significantly faster timescales Supplementary Fig. 6. As expected, the distinct distribution of 


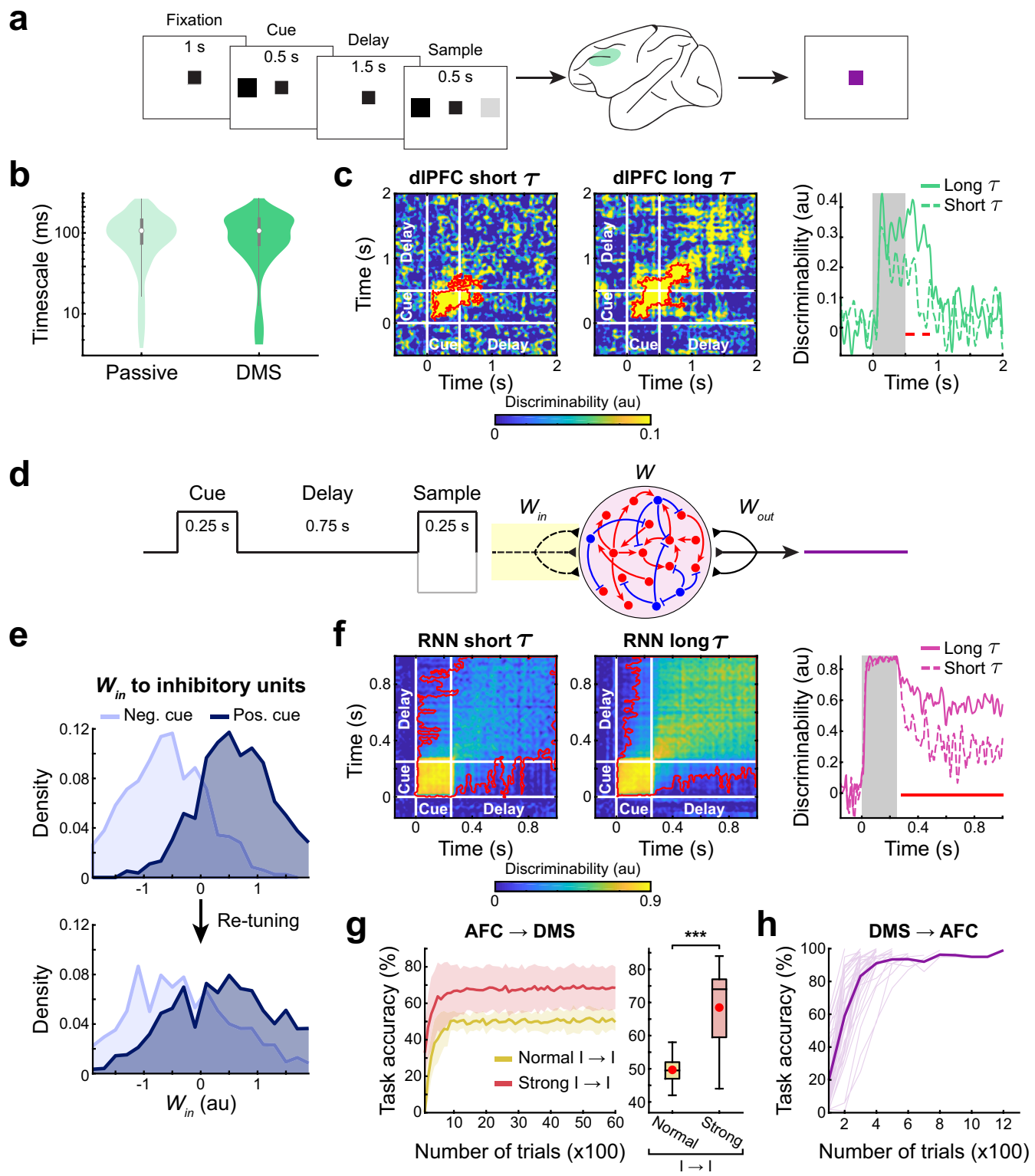

Fig. 8| Strong $I \rightarrow I$ connections intrinsic to prefrontal cortex. a, Passive task paradigm used by Constantinidis et al. 18 to train the same four monkeys before they learned the DMS tasks (Fig. 1 p). b, Distribution of the neuronal timescales from the monkeys before (i.e., passive) and after they learned the DMS tasks. c, Cross-temporal decoding matrices and within-time decoding timecourses from the short and long $\tau$ subgroups. d, Passive task paradigm used to re-train our DMS RNNs. Only the input weights (dashed lines with yellow shading) were trained. e, Distribution of the input weights projecting to the two inhibitory subgroups tuned to the two cue stimuli from all 40 DMS RNNs before (top) and after (bottom) retraining. f, Cross-temporal decoding matrices and within-time decoding timecourses from the short and long $\tau$ subgroups for the re-trained DMS RNNs. g, Task performance during re-training of the AFC rate RNNs to perform the DMS task (left) and average performance at the end of training (right). The task performance significantly increased when $I \rightarrow I$ connections were strengthened (orange; see Methods). Shaded area, \pm s.d. $\mathbf{h}$, Task performance during re-training of the DMS rate RNNs to perform the AFC task. Individual networks (light) and mean across 40 DMS RNNs (bold). Boxplot central lines, median; red circles, mean; bottom and top edges, lower and upper quartiles; whiskers, 1.5 *interquartile range; outliers not plotted. Red contours indicate significant discriminability (cluster-based permutation test, $P<0.05$; see Methods). Red lines indicate significant differences in decoding between the short and long $\tau$ groups (cluster-based permutation test, $P<0.05$; see Methods). ${ }^{* * *} P<0.0001$ by Wilcoxon signed-rank test. 
250

251

252 the cross-temporal discriminability analysis on the re-trained RNNs showed that the cue stimulus

253 information during the delay period was not maintained as robustly by long $\tau$ units (Fig. 8f).

254 However, the long $\tau$ units still carried significantly higher information than the short $\tau$ units

255 throughout the delay window.

256 The above results from the experimental data and our model strongly suggest that higher 257 cortical areas might have intrinsically diverse and robust inhibitory signaling. This innate property,

258 in turn, would give rise to long neuronal timescales, and the incoming connections to these areas 259 could undergo plastic changes to support various higher cognitive functions that require integration 260 of information on a slower timescale. Along this line of thought, we hypothesized that the AFC 261 RNNs, which do not have strong inhibitory-to-inhibitory signaling, are not capable of performing 262 WM tasks by simply re-tuning the input weights only. With the recurrent architecture $(W)$ fixed, 263 we attempted to re-train the input weights of the 40 AFC RNNs to perform the DMS task, but 264 none of the networks could be trained successfully (yellow line in Fig. 8s). When we repeated 265 the re-training procedure with the $I \rightarrow I$ recurrent connections strengthened (see Methods), the 266 performance of the AFC RNNs significantly improved (magenta line in Fig. 8s). On the other 267 hand, the input weights of the DMS RNNs could be successfully tuned to perform the AFC task 268 (Fig. 8h), further confirming the hierarchical organization of these two RNN models.

\section{Discussion}

270 In this study, we provide a computational model that gives rise to task-specific spontaneous tempo271 ral dynamics, reminiscent of the hierarchy of neuronal timescales observed across primate cortical 272 areas [1]. When trained on a WM task, our RNN model was composed of units with long timescales 273 whose distribution was surprisingly similar to the one obtained from the primate dlPFC. In addi274 tion, the long-timescale units encoded and maintained WM-related information more robustly than 275 the short-timescale units during the delay period. By analyzing the connectivity structure of the 276 model, we showed that a unique circuit motif that incorporates strong $I \rightarrow I$ synapses is an inte277 gral component for WM computations and slow baseline temporal properties. Interestingly, $I \rightarrow I$ 278 synaptic weights could be manipulated to control both task performance and neuronal timescales 
279

280

tightly. Our work also provides mechanistic insight into how $I \rightarrow I$ connectivity supports memory storage and dynamic baseline activity patterns crucial for long neuronal timescales. Lastly, we propose that the microcircuitry we identified is intrinsic to higher-order cortical areas enabling them to perform cognitive tasks that require steady integration of information.

Relating specific baseline spiking activities to the underlying circuit mechanisms has been challenging partly due to the lack of computational models capable of both performing cognitive tasks and capturing temporal dynamics derived from experiments. Although it is possible to use continuous rate RNN models, which have been widely used to uncover neural mechanisms behind cognitive processes 25 29], to study neuronal timescales, our spiking RNN model allowed us to (1) use the same experimental procedures previously used to estimate neuronal timescales, (2) easily interpret and compare our model results with experimental findings, and (3) uncover spiking statistics (spike-count Fano factors) associated with long neuronal timescales.

Our work revealed that strong $I \rightarrow I$ connections are critical for long neuronal timescales, and we investigated the functional implication of such connections in WM-related behavior. Despite the fact that excitatory pyramidal cells make up the majority of neurons in cortical areas, inhibitory interneurons have been shown to exert greater influence at the local network level [30, 31]. Furthermore, different subtypes of interneurons play functionally distinct roles in cortical computations 9 , 14. In agreement with these observations, recent studies uncovered the importance of disinhibitory gating imposed by VIP interneurons $10,13,32,33$. Through inhibition of SST and PV neurons, VIP interneurons have a unique ability to disinhibit pyramidal cells and create "holes" in a dense "blanket of inhibition" 33. Surprisingly, optogenetically activating VIP neurons in the PFC of mice trained to perform a WM task significantly enhanced their task performance highlighting that disinhibitory signaling is vital for memory formation and recall [10]. Similar to VIP neurons, SST interneurons have also been shown to disinhibit excitatory cells for fear memory [11, 12. Intriguingly, the connectivity structures of the RNNs we trained on a WM task using supervised learning also centered around disinhibitory circuitry with strong $I \rightarrow I$ synapses (Fig. 6). The strength of the $I \rightarrow I$ connections was tightly coupled to the task performance of the RNNs. Thus, our work suggests that microcircuitry specializing in disinhibition could be a common substrate in

7 higher-order cortical areas that require short-term memory maintenance. 
Most notably, our results shed light on exactly how robust $I \rightarrow I$ connections maintain stable memory storage and long neuronal timescales. By dissecting our WM RNN model, we found that strong mutual inhibition between two oppositely-tuned inhibitory subgroups was necessary for

311 maintaining stimulus-specific information during the delay period (Fig. 6). We also illustrated that

312 our model units that were strongly modulated by $I \rightarrow I$ synapses displayed highly dynamic baseline

313 activities leading to both large trial-to-trial Fano factors and long neuronal timescales (Fig. 7). For

314 the neural data, it was not possible to identify neurons tightly regulated by inhibitory synapses, as 315 connectivity patterns are challenging to infer from firing activities alone. However, we showed that 316 long $\tau$ neurons in the primate PFC also exhibited high trial-to-trial variability. Thus, our findings suggest that spontaneous variability or Fano factors could be a good indicator of the underlying 318 circuit mechanisms: neurons with asynchronously occurring synchronous firing patterns (i.e., high 319 variability) could make up WM-related microcircuits.

Cognitive flexibility is one of the hallmarks of the prefrontal cortex [34, 35]. If higher-order 321 areas are indeed wired with specific and robust $I \rightarrow I$ synapses that give rise to stable temporal 322 receptive fields, then what would happen to these connections during learning? Would learning 323 a new task disrupt the existing $I \rightarrow I$ connectivity structure, thereby abolishing the previously 324 established timescale distribution? To answer these questions, we analyzed neuronal timescales 325 from monkeys before and after they learned a WM task. The distribution of the timescales was 326 not significantly different between the two conditions, suggesting that learning the WM task did 327 not perturb the intrinsic temporal structure in the dlPFC (Fig. 8). To test if a network is flexible 328 enough to perform other tasks with its timescale dynamics fixed, we re-trained our WM model with 329 its recurrent connectivity frozen to perform a non-WM task (Fig. 8). With re-tuning only the input 330 connections, the WM RNNs could be easily re-trained to perform the non-WM task, implying that 331 these networks are flexible to perform tasks that require not only long timescales but also short 332 timescales. In contrast, RNNs trained on the non-WM task could not be re-trained to perform the 333 WM task. Thus, our work suggests that disinhibitory microcircuits with strong $I \rightarrow I$ synapses 334 could give rise to a flexible module capable of performing a wide range of tasks.

335 Although our model can capture several experimental findings, a few interesting questions re336 main for future studies. For example, our spiking RNN model utilizes connectivity patterns derived 
337 from a gradient-descent approach, which is not biologically plausible. It will be important to char338 acterize if more biologically valid learning mechanisms, such as reinforcement learning or Hebbian 339 learning, also generate spiking networks with heterogeneous neuronal timescales. Another unex340 plored aspect is nonlinear dendritic computations. SST interneurons are known for targeting den341 drites of pyramidal cells, and such dendritic inhibition has been associated with gating information 342 36. Incorporating dendritic processes into our model could elucidate the computational benefits of 343 dendritic inhibition over perisomatic inhibition during WM. In summary, we have explored a neu344 ral circuit mechanism that performs logical computations over time with stable temporal receptive 345 fields. 
346

347

348

349

350

351

353

354

355

356

357

358

359

360

361

362

363

364

365

366

367

\section{References}

1. Murray, J. D. et al. A hierarchy of intrinsic timescales across primate cortex. Nature neuroscience 17, 1661-1663 (2014).

2. Chaudhuri, R., Knoblauch, K., Gariel, M.-A., Kennedy, H. \& Wang, X.-J. A large-scale circuit mechanism for hierarchical dynamical processing in the primate cortex. Neuron $\mathbf{8 8 , 4 1 9 - 4 3 1}$ (2015).

3. Cavanagh, S. E., Wallis, J. D., Kennerley, S. W. \& Hunt, L. T. Autocorrelation structure at rest predicts value correlates of single neurons during reward-guided choice. Elife 5, e18937 (2016).

4. Miller, E. K., Erickson, C. A. \& Desimone, R. Neural mechanisms of visual working memory in prefrontal cortex of the macaque. Journal of neuroscience 16, 5154-5167 (1996).

5. Fuster, J. M. \& Alexander, G. E. Neuron activity related to short-term memory. Science 173, $652-654$ (1971).

6. Fascianelli, V., Tsujimoto, S., Marcos, E. \& Genovesio, A. Autocorrelation Structure in the Macaque Dorsolateral, But not Orbital or Polar, Prefrontal Cortex Predicts Response-Coding Strength in a Visually Cued Strategy Task. Cerebral cortex 29, 230-241 (2017).

7. Cavanagh, S. E., Towers, J. P., Wallis, J. D., Hunt, L. T. \& Kennerley, S. W. Reconciling persistent and dynamic hypotheses of working memory coding in prefrontal cortex. Nature communications 9 (2018).

8. Wasmuht, D. F., Spaak, E., Buschman, T. J., Miller, E. K. \& Stokes, M. G. Intrinsic neuronal dynamics predict distinct functional roles during working memory. Nature communications $\mathbf{9}$, 3499 (2018).

9. Kim, D. et al. Distinct roles of parvalbumin- and somatostatin-expressing interneurons in working memory. Neuron 92, $902-915$ (2016).

10. Kamigaki, T. \& Dan, Y. Delay activity of specific prefrontal interneuron subtypes modulates memory-guided behavior. Nature neuroscience 20, 854-863 (2017).

11. $\mathrm{Xu}, \mathrm{H}$. et al. A disinhibitory microcircuit mediates conditioned social fear in the prefrontal cortex. Neuron 102, 668-682 (2019). 
374 12. Cummings, K. A. \& Clem, R. L. Prefrontal somatostatin interneurons encode fear memory. Nature neuroscience 23, 61-74 (2019).

376 13. Krabbe, S. et al. Adaptive disinhibitory gating by VIP interneurons permits associative learning. Nature neuroscience 22, 1834-1843 (2019).

378

379

380

381

382

383

384

385

386

14. Pfeffer, C. K., Xue, M., He, M., Huang, Z. J. \& Scanziani, M. Inhibition of inhibition in visual cortex: the logic of connections between molecularly distinct interneurons. Nature neuroscience 16, 1068-1076 (2013).

15. Tremblay, R., Lee, S. \& Rudy, B. GABAergic interneurons in the neocortex: from cellular properties to circuits. Neuron 91, 260-292 (2016).

16. Medalla, M., Gilman, J. P., Wang, J.-Y. \& Luebke, J. I. Strength and diversity of inhibitory signaling differentiates primate anterior cingulate from lateral prefrontal cortex. Journal of neuroscience 37, 4717-4734 (2017).

17. Mongillo, G., Rumpel, S. \& Loewenstein, Y. Inhibitory connectivity defines the realm of excitatory plasticity. Nature neuroscience 21, 1463-1470 (2018).

18. Constantinidis, C., Qi, X.-L. \& Meyer, T. Single-neuron spike train recordings from macaque prefrontal cortex during a visual working memory task before and after training. Crcns.org (2016).

19. Kim, R., Li, Y. \& Sejnowski, T. J. Simple framework for constructing functional spiking recurrent neural networks. Proceedings of the national academy of sciences 116, 22811-22820 (2019).

20. Qi, X.-L., Meyer, T., Stanford, T. R. \& Constantinidis, C. Changes in prefrontal neuronal activity after learning to perform a spatial working memory task. Cerebral cortex 21, 27222732 (Apr. 2011).

21. Meyer, T., Qi, X.-L., Stanford, T. R. \& Constantinidis, C. Stimulus selectivity in dorsal and ventral prefrontal cortex after training in working memory tasks. Journal of neuroscience 31, 6266-6276 (2011).

22. Stokes, M. G. et al. Dynamic coding for cognitive control in prefrontal cortex. Neuron $\mathbf{7 8 ,}$ 364-375 (2013). 
402 23. Spaak, E., Watanabe, K., Funahashi, S. \& Stokes, M. G. Stable and dynamic coding for 403 working memory in primate prefrontal cortex. Journal of neuroscience 37, 6503-6516 (2017).

404 24. Meyer, T., Qi, X.-L. \& Constantinidis, C. Persistent discharges in the prefrontal cortex of 405 monkeys naïve to working memory tasks. Cerebral cortex 17, i70-i76 (2007).

406 25. Mante, V., Sussillo, D., Shenoy, K. V. \& Newsome, W. T. Context-dependent computation 407 by recurrent dynamics in prefrontal cortex. Nature 503, 78-84 (2013).

408 26. Song, H. F., Yang, G. R. \& Wang, X.-J. Training excitatory-inhibitory recurrent neural networks for cognitive tasks: a simple and flexible framework. Plos computational biology 12, e1004792 (2016).

411 27. Miconi, T. Biologically plausible learning in recurrent neural networks reproduces neural dy412 namics observed during cognitive tasks. Elife 6, e20899 (2017).

413 28. Orhan, A. E. \& Ma, W. J. A diverse range of factors affect the nature of neural representations underlying short-term memory. Nature neuroscience 22, 275-283 (2019).

415 29. Yang, G. R., Joglekar, M. R., Song, H. F., Newsome, W. T. \& Wang, X.-J. Task representations in neural networks trained to perform many cognitive tasks. Nature neuroscience 22, 297-306 (2019).

30. Kepecs, A. \& Fishell, G. Interneuron cell types are fit to function. Nature 505, 318-326 (2014).

31. Batista-Brito, R. et al. Developmental dysfunction of VIP interneurons impairs cortical circuits. Neuron 95, 884 -895.e9 (2017).

32. Pi, H.-J. et al. Cortical interneurons that specialize in disinhibitory control. Nature 503, 521$524(2013)$.

33. Karnani, M. M. et al. Opening holes in the blanket of inhibition: localized lateral disinhibition by VIP interneurons. Journal of neuroscience 36, 3471-3480 (2016). of neuroscience 24, 167-202 (2001). lanta, 2011). 
429 36. Yang, G. R., Murray, J. D. \& Wang, X.-J. A dendritic disinhibitory circuit mechanism for $430 \quad$ pathway-specific gating. Nature communications 7 (2016).

431 37. Maris, E. \& Oostenveld, R. Nonparametric statistical testing of EEG- and MEG-data. Journal $432 \quad$ of neuroscience methods 164, $177-190$ (2007).

\section{Acknowledgements}

434 We are grateful to Ben Tsuda, Yusi Chen, and Jason Fleischer for helpful discussions and feedback 435 on the manuscript. We also thank Jorge Aldana for assistance with computing resources. This 436 work was funded by the National Institute of Mental Health (F30MH115605-01A1 to R.K.). We 437 also gratefully acknowledge the support of NVIDIA Corporation with the donation of the Quadro 438 P6000 GPU used for this research. The funders had no role in study design, data collection and 439 analysis, decision to publish, or preparation of the manuscript.

\section{Author contributions}

441 R.K. and T.J.S. designed the study and wrote the manuscript. R.K. performed the analyses and 442 simulations.

\section{Declaration of interests}

444 The authors declare no competing interests. 


\section{Methods}

446 Continuous rate RNN model. The spiking RNNs used in the main text were generated by

447 first training their counterpart continuous-variable rate RNNs using a gradient descent algorithm.

448 After training, the continuous RNNs were converted to leaky integrate-and-fire (LIF) RNNs using

449 the method that we previously developed 19. The continuous RNN model contained $N=200$

450 recurrently connected units that were governed by

$$
\begin{aligned}
\boldsymbol{\tau}^{d} \frac{d \boldsymbol{x}}{d t} & =-\boldsymbol{x}+W^{\text {rate }} \boldsymbol{r}^{\text {rate }}+\boldsymbol{I}_{\text {ext }} \\
\boldsymbol{r}^{\text {rate }} & =\frac{1}{1+\exp (-\boldsymbol{x})}
\end{aligned}
$$

451 where $20 \mathrm{~ms} \leq \boldsymbol{\tau}^{\boldsymbol{d}} \leq 125 \mathrm{~ms} \in \mathbb{R}^{1 \times N}$ corresponds to the synaptic decay time constants for the $N$

452 units in the network, $\boldsymbol{x} \in \mathbb{R}^{1 \times N}$ is the synaptic current variable, $W^{\text {rate }} \in \mathbb{R}^{N \times N}$ is the synaptic

453 connectivity matrix, and $\boldsymbol{r}^{\text {rate }} \in \mathbb{R}^{1 \times N}$ refers to the firing rate estimates of the units. A standard

454 logistic sigmoid function was used to estimate a firing rate of a neuron from its synaptic current $455(x)$.

456 The external currents $\left(\boldsymbol{I}_{\text {ext }}\right)$ include task-specific input stimulus signals (see Training details) 457 along with a Gaussian white noise variable:

$$
\boldsymbol{I}_{e x t}=W_{i n} \boldsymbol{u}+\mathcal{N}(0,0.01)
$$

458 where the time-varying, task-specific stimulus signals $\left(\boldsymbol{u} \in \mathbb{R}^{N_{i n} \times 1}\right)$ are given to the network via $459 W_{\text {in }} \in \mathbb{R}^{N \times N_{\text {in }}}$, a Gaussian random matrix with zero mean and unit variance. $N_{\text {in }}$ corresponds to 460 the number of input signals associated with a specific task, and $\mathcal{N}(0,0.01) \in \mathbb{R}^{N \times 1}$ represents a 461 Gaussian random noise with zero mean and variance of 0.01 .

462 A linear readout of the population activity was used to define the output of the rate network:

$$
o^{\text {rate }}(t)=W_{\text {out }}^{\text {rate }} \boldsymbol{r}^{\text {rate }}(t)
$$

463 where $W_{\text {out }}^{\text {rate }} \in \mathbb{R}^{1 \times N}$ refers to the readout weights. 
Eq. (1) is discretized using the first-order Euler approximation method:

$$
\begin{aligned}
\boldsymbol{x}_{t} & =\left(1-\frac{\Delta t}{\boldsymbol{\tau}^{\boldsymbol{d}}}\right) \boldsymbol{x}_{t-1}+\frac{\Delta t}{\boldsymbol{\tau}^{\boldsymbol{d}}}\left(W^{\text {rate }} \boldsymbol{r}_{t-1}^{\text {rate }}+W_{i n} \boldsymbol{u}_{t-1}\right) \\
& +\mathcal{N}(0,0.01)
\end{aligned}
$$

465

466

where $\Delta t=5 \mathrm{~ms}$ is the discretization time step size used throughout this study.

Training details. Adam (adaptive moment estimation), a stochastic gradient descent algorithm, was used to update the synaptic decay variable $\left(\tau_{s}\right)$, recurrent connections $\left(W^{\text {rate }}\right)$ and readout weights $\left(W_{\text {out }}^{\text {rate }}\right)$. The learning rate was set to 0.01 , and the TensorFlow default values were used for the first and second moment decay rates. In addition, Dale's principle (i.e., separate excitatory and inhibitory populations) was imposed using the method previously proposed [26]. For re-training previously trained RNNs (Fig. 8), only the input weights $\left(W_{i n}\right)$ were trainable, and the recurrent weights and the readout weights were fixed to their trained values.

Two LIF RNN models were employed in this study by training rate RNNs on two different tasks: delayed match-to-sample (DMS) and 2-alternative forced choice (AFC) tasks.

$D M S R N N s$. For the DMS RNN model, the input matrix $\left(\boldsymbol{u} \in \mathbb{R}^{2 \times 500}\right)$ contained two input channels for two sequential stimuli (over 500 time steps with $5 \mathrm{~ms}$ step size). The first channel delivered the first stimulus (250 ms in duration) after $1 \mathrm{~s}$ (200 time steps) of fixation, while the second channel modeled the second stimulus (250 ms in duration), which began $50 \mathrm{~ms}$ after the offset of the first stimulus. The short delay $(50 \mathrm{~ms})$ allowed rate RNNs to learn the task efficiently, and the delay duration was increased after training (see below). During each stimulus window, the corresponding input channel was set to either -1 or +1 . If the two sequential stimuli had the same sign $(-1 /-1$ or $+1 /+1)$, the network was trained to produce an output signal approaching +1 after the offset of the second stimulus. If the stimuli had opposite signs $(-1 /+1$ or $+1 /-1)$, then the network produced an output signal approaching -1. The training was stopped when the loss function fell below 7 , and the task performance was greater than 95\%. After the rate RNNs were successfully trained and converted to LIF networks, a subgroup of LIF RNNs that performed the actual DMS paradigm 87 used in the main text (i.e., delay duration set to $750 \mathrm{~ms}$ ) with accuracy greater than $95 \%$ were identified and analyzed. For Figs. 5, 6 and 7, a group of LIF RNNs that performed the DMS task 
489 with accuracy between $60 \%$ and $80 \%$ was used.

490 AFC RNNs. The input matrix $\left(\boldsymbol{u} \in \mathbb{R}^{1 \times 350}\right)$ for the AFC paradigm was set to 0 for the first 200

491 time steps (i.e., $1 \mathrm{~s}$ fixation). A short stimulus (125 ms in duration) of either -1 or +1 was given

492 after the fixation period. After the stimulus offset, the network was trained to produce an output

493 signal approaching -1 for the " -1 " stimulus and +1 for the " +1 " stimulus. The training termination

494 criteria were the same as those used for the DMS model above.

495 Spiking RNN model. For our spiking RNN model, we considered a network of leaky integrate496 and-fire (LIF) units recurrently connected to one another. These units are governed by:

$$
\tau_{m} \frac{d v_{i}(t)}{d t}=-v_{i}(t)+\left(x_{i}(t)+I_{e x t}(t)\right) R
$$

497 where $\tau_{m}$ is the membrane time constant $(10 \mathrm{~ms}), v_{i}(t)$ is the membrane voltage of unit $i$ at 498 time $t, x_{i}(t)$ is the synaptic input current that unit $i$ receives at time $t, I_{e x t}$ is the external input 499 current, and $R$ is the leak resistance (set to 1 ). The synaptic input current $(x)$ is modeled using a 500 double-exponential synaptic filter applied to the presynaptic spike trains:

$$
\begin{aligned}
x_{i} & =\sum_{j=1}^{N} W_{i j}^{s p k} r_{j}^{s p k} \\
\frac{d r_{i}^{s p k}}{d t} & =-\frac{r_{i}^{s p k}}{\tau_{i}^{d}}+s_{i} \\
\frac{d s_{i}}{d t} & =-\frac{s_{i}}{\tau_{r}}+\frac{1}{\tau_{r} \tau_{i}^{d}} \sum_{t_{i}^{k}<t} \delta\left(t-t_{i}^{k}\right)
\end{aligned}
$$

501 where $W_{i j}^{s p k}$ is the recurrent connection strength from unit $j$ to unit $i, \tau_{r}=2 \mathrm{~ms}$ is the synaptic 502 rise time and $\tau_{i}^{d}$ refers to the synaptic decay time for unit $i$. The synaptic decay time constant 503 values and the recurrent connectivity matrix were transferred from the trained rate RNNs (more 504 details described in 19]). The spike train produced by unit $i$ is represented as a sum of Dirac $\delta$ 505 functions, and $t_{i}^{k}$ refers to the $k$-th spike emitted by unit $i$.

506 The external current input $\left(I_{\text {ext }}\right)$ contained task-specific input values along with a constant 507 background current set near the action potential threshold. The output of our spiking model at 
time $t$ is given by

$$
o^{s p k}(t)=W_{o u t}^{s p k} \boldsymbol{r}^{s p k}(t)
$$

509 where the readout weights $\left(W_{\text {out }}^{\text {spk }}\right)$ are also transferred from the trained rate RNN model.

510 Other LIF model parameters included the action potential threshold $(-40 \mathrm{mV})$, the reset po511 tential $(-65 \mathrm{mV})$, the absolute refractory period $(2 \mathrm{~ms})$, and the constant bias current $(-40 \mathrm{pA})$. 512 Eq. (2) was discretized using a first-order Euler method with $\Delta t=0.05 \mathrm{~ms}$.

513 Electrophysiological recordings. Extracellular recordings, previously published and described 514 in detail [18, 20, 21, were analyzed to validate our RNN model. The dataset contained spike515 train recordings from four rhesus macaque monkeys before and after they learned two DMS tasks. 516 Briefly, for the pre-training condition, the monkeys were rewarded for maintaining fixation on the 517 center of the screen regardless of the visual stimuli shown throughout the trial (Fig. 8a). For the 518 post-training condition, the monkeys were trained on two DMS tasks: spatial and feature DMS 519 tasks. For the spatial task (Fig. 1b), the monkeys were trained to report if two sequential stimuli 520 matched in their spatial locations. For the feature task, they had to distinguish if two sequential 521 stimuli matched in their shapes. The dataset included spike times from single neurons in the dorsal 522 and ventral PFC, but only the units from the dorsal PFC were analyzed for this study.

523 Estimation of neuronal timescales. To estimate neuronal timescales, we computed the decay 524 time constant of the spike-count autocorrelation function for each unit during the fixation period 525 1]. For each unit, we first binned its spike trains during the fixation period over multiple trials using 526 a non-overlapping 50-ms moving window. Since the fixation duration was $1 \mathrm{~s}$ for the experimental 527 data and our model, this resulted in a [Number of Trials $\times 20$ ] spike-count matrix for each unit. 528 For the experimental data, the minimum number of trials required for a neuron to be considered 529 for analysis was 11 trials. The average number of trials from all the neurons from the post-training 530 condition was $86.8 \pm 35.1$ (mean \pm s.d.) trials. For the pre-training condition, the average number 531 of trials was $95.4 \pm 44.4$. For the RNN model, we generated 50 trials for each unit.

532 Next, Pearson's correlation coefficient $(\rho)$ was computed between two time bins (i.e., two 533 columns in the spike-count matrix) separated by a lag $(\Delta)$. The coefficient was calculated for 534 all possible pairs with a maximum lag of $600 \mathrm{~ms}$. The coefficients were averaged for each lag value, 
535 and an exponential decay function was fitted across the average coefficient values $(\bar{\rho})$ using the

536 Levenberg-Marquardt nonlinear least-squares method:

$$
\bar{\rho}(\Delta)=A\left(\exp \left(-\frac{\Delta}{\tau}\right)+B\right)
$$

537 where $A$ and $B$ are the amplitude and the offset of the fit, respectively. The timescale $(\tau)$ defines

538 how fast the autocorrelation decays and was used to estimate each neuron's timescale.

539 The following inclusion criteria (commonly used in previous experimental studies) were applied

540 to the RNN model and the experimental data: (1) minimum average firing rate of $1 \mathrm{~Hz}$ during the

541 fixation period for the experimental data and $2.5 \mathrm{~Hz}$ for the RNN model, (2) $0<\tau \leq 500 \mathrm{~ms}$, (3)

$542 A>0$, and (4) a first decrease in $\rho$ earlier than $\Delta=150 \mathrm{~ms}$. In addition, the fitting was started

543 after the first decrease in autocorrelation. For the experimental dataset, 325 dlPFC units from the

544 post-training condition and 434 units from the pre-training condition satisfied the above criteria.

545 For the DMS RNN model, 931 units from 40 good performance RNNs and 604 units from 26 poor

546 performance RNNs met the criteria. For the AFC model, 1138 units from 40 RNNs satisfied the

547 criteria.

548 Cross-temporal decoding analysis. The amount of information encoded by each unit was esti-

549 mated using cross-temporal decoding analysis [8, 22, 23]. For both experimental and model data,

550 a Gaussian kernel (s.d. $=50 \mathrm{~ms}$ ) was first applied to the spike-trains to obtain the firing rate

551 estimates over time. For each cue stimulus identity, each neuron's firing rate timecourses were

552 divided into two splits (even vs. odd trials) and averaged across trials within each split. There

553 were 9 cue conditions (i.e., 9 spatial locations) for the spatial DMS task and 8 cue conditions (i.e.,

5548 shapes) for the feature DMS task. Within each task, all possible pairwise differences in mean

555 firing rates between any two cue conditions for each neuron in each split were computed. Next,

556 Pearson's correlation coefficient was determined for each pairwise difference condition between the

557 two splits (at each time point across neurons). The correlation coefficients from both tasks (36

558 pairwise difference conditions for the spatial task and 28 conditions for the feature task) at each

559 time point were averaged after applying the Fisher's z-transformation resulting in a single measure

560 we refer to as a discriminability or decodability score. The within-time discriminability scores were 
561

562

563

564

565

566

567

568

569

570

571

572

573

574

575

576

577

578

579

580

581

582

583

584

585

586

587

588

589

computed from the correlation coefficients at $t_{1}=t_{2}$ where $t_{1}$ and $t_{2}$ refer to the time points used for the two splits.

Nonparametric cluster-based permutation tests were utilized to account for multiple comparisons and to determine significant discriminability (Fig. 3a) and differences in discriminability between short and long $\tau$ subgroups (Figs. 3 and 8) 37. To identify significant clusters in the cross-temporal matrices (Fig. 3 and Fig. 8k,f), cue stimulus condition labels were randomly shuffled for 1,000 times within each split to construct the null distribution. A point was considered significant if its value exceeded the 95th percentile of the null distribution, and the largest cluster size (i.e., number of contiguous points that were significant) from the data was compared against the null distribution of the largest cluster size values to correct for multiple comparisons. To determine if within-time decoding timecourses were significantly different between long and short $\tau$ groups (Fig. 3b and Fig. 8k,f), $\tau$ group labels were randomly shuffled for 1,000 times within each split and each task. Again, a time point was considered significant if it was greater than the 95th percentile of the null distribution. Similar multiple comparison correction, as described above, was applied.

Cross-temporal decoding matrices and within-time decoding timecourses for the dlPFC data Figs. 3 and 8 ) were smoothed for better visualization, but all statistical tests were performed on unsmoothed data.

Connectivity rewiring method. For Fig. 4 , we characterized which connection type contributed the most to the long neuronal timescales observed in the DMS RNN model by randomly shuffling connections belonging to each type $(I \rightarrow I, I \rightarrow E, E \rightarrow I$, or $E \rightarrow E)$ while preserving the original distribution of the connection types. For the $I \rightarrow I$ type, all the outward connections from each inhibitory unit to other inhibitory units were first identified. These connections were then rewired randomly in a manner that preserved their connection identity (i.e., $I \rightarrow I$ ). This procedure was repeated for the other three synaptic types. For Fig. 5, all the synaptic weights corresponding to each connection type were either decreased or increased by $30 \%$ without rewiring.

To quantify the amount of cue-specific information maintained during the delay period in each of the four shuffling conditions (Fig. 4f), we performed the within-time decoding analysis (see above)

58 for all the units in each RNN per shuffling condition. This resulted in 40 within-time decoding 
590 timecourses (one for each RNN) for each rewiring condition.

591 Cue stimulus selectivity. In order to identify inhibitory units selective for each of the two cue

592 stimuli $(-1$ or +1$)$, we computed a cue preference index $(\theta)$ for each unit using:

$$
\theta_{i}=\frac{r_{i,+1}-r_{i,-1}}{r_{i,+1}+r_{i,-1}}
$$

593 where $r_{i,+1}$ refers to the average firing rate of unit $i$ across positive cue stimulus trials (50 trials)

594 during the cue stimulus window, while $r_{i,-1}$ indicates the average activity across negative cue 595 stimulus trials (50 trials). Thus, $\theta_{i}>0$ indicates that unit $i$ prefers the positive cue stimulus over 596 the negative stimulus. Based on this selectivity measure, two subgroups of inhibitory units (one 597 for $\theta>0$ and the other for $\theta<0$ ) were identified for each DMS RNN.

598 Spike-count Fano factors. The relationship between spike-count variability and neuronal timescales 599 was investigated by computing trial-to-trial spike-count Fano factors during the fixation period 600 (Fig. 7). For each unit included in the timescale analysis, the variance of the total number of 601 spikes within the 1-s fixation window across trials was first computed. The Fano factor was then 602 calculated by dividing the variance by the mean spike count. The trials used for computing the 603 Fano factors were identical as those used for estimating the neuronal timescales for both neural 604 and RNN data.

605 Reconfiguring pre-trained RNNs. In Fig. 8g, h, the continuous-variable rate RNNs trained to 606 perform the AFC and DMS tasks were used. For Fig. 8s, only the input weights $\left(W_{i n}\right)$ for the 607 AFC RNNs were re-trained via the same gradient descent algorithm to perform the DMS task. 608 The $I \rightarrow I$ connections were either unaltered (yellow in Fig. 8p) or increased by 200\% (orange 609 in Fig. 8 ). In Fig. 8h, only the input weights for the DMS RNNs were reconfigured to perform 610 the AFC task. The maximum number of training trials was set to 6,000 trials for computational 611 efficiency.

\section{Code availability}

613 The code for the analyses performed in this work will be made available at https://github.com/ 614 rkim35/wmRNN 
bioRxiv preprint doi: https://doi.org/10.1101/2020.02.11.944751; this version posted February 12,2020 . The copyright holder for this preprint (which was not certified by peer review) is the author/funder, who has granted bioRxiv a license to display the preprint in perpetuity. It is made available under aCC-BY-NC-ND 4.0 International license.

\section{Data availability}

616 The trained RNN models used in the present study will be available as MATLAB-formatted data

617 at https://github.com/rkim35/wmRNN 


\section{Supplementary Figures}

a

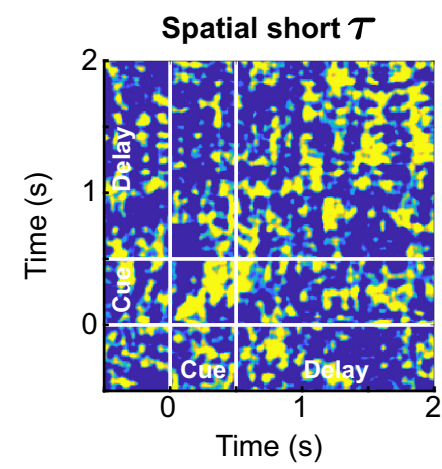

b

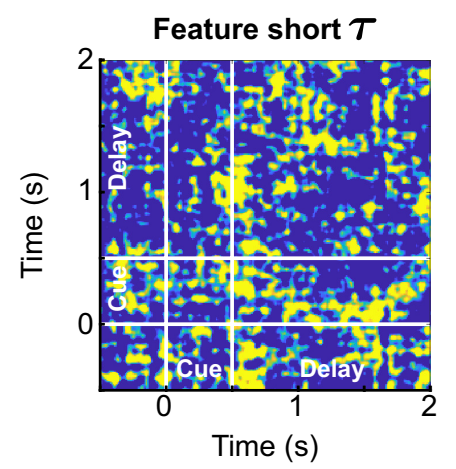

Spatial long $\tau$

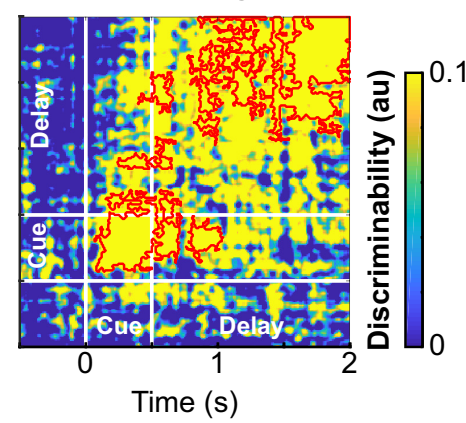

Feature long $\tau$

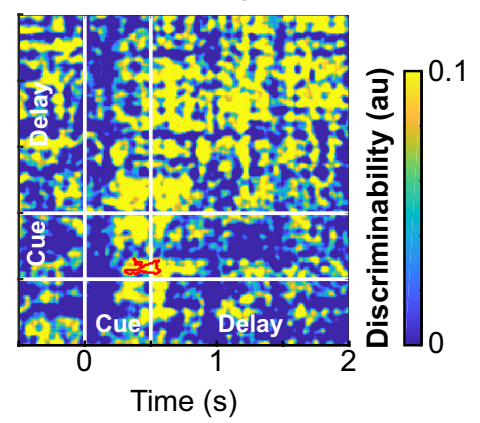

Spatial
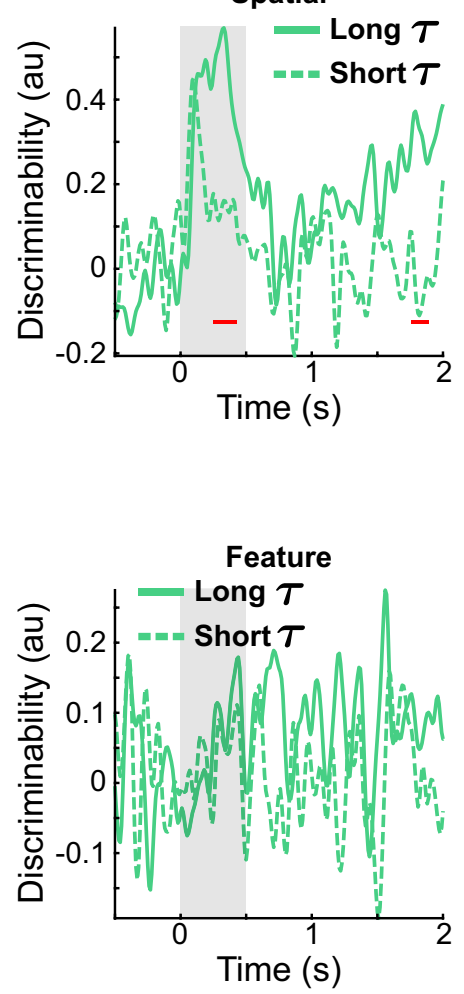

Supplementary Fig. 1 | Long $\tau$ units maintain cue stimulus information during the delay period of the spatial DMS task. a, Cross-temporal discriminability matrices and the within-time decoding timecourses from the short and long $\tau$ groups of the dlPFC data limited to the spatial DMS task. b, Cross-temporal discriminability matrices and the within-time decoding timecourses from the short and long $\tau$ groups of the dIPFC data limited to the feature DMS task. Gray shading, cue stimulus window. Red contours indicate significant decodability (see Methods; cluster-based permutation test, $P<0.05$ ). Red lines indicate significant differences in decoding between the short and long $\tau$ groups (see Methods; cluster-based permutation test, $P<0.05)$. 
a

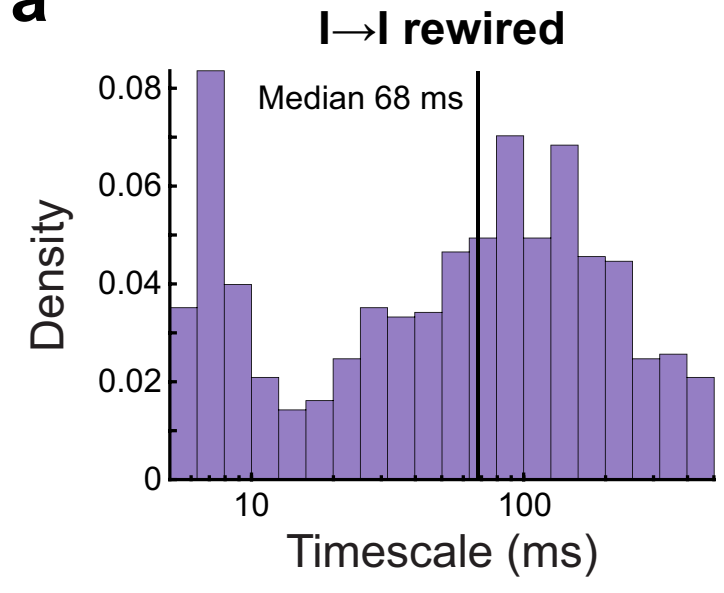

C

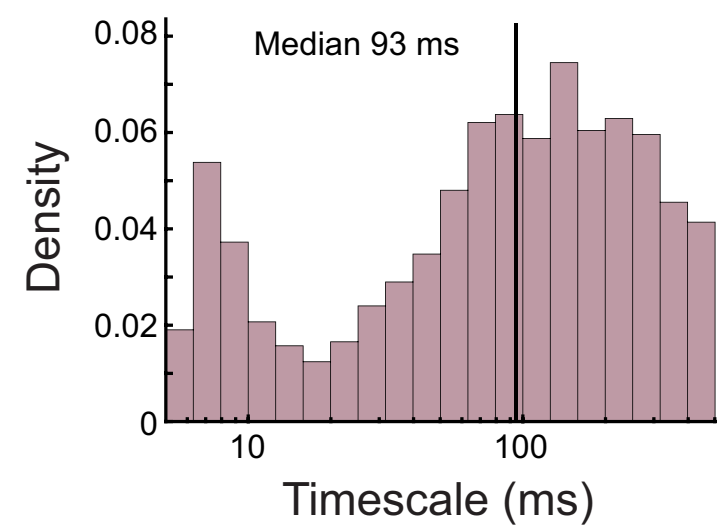

b

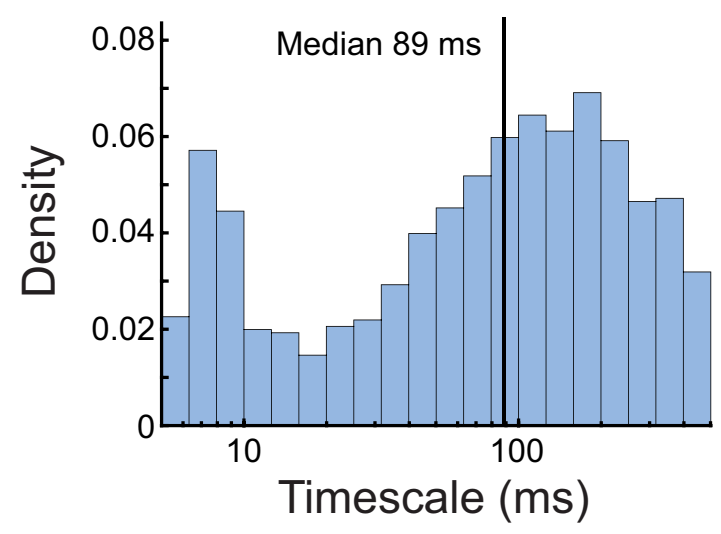

d

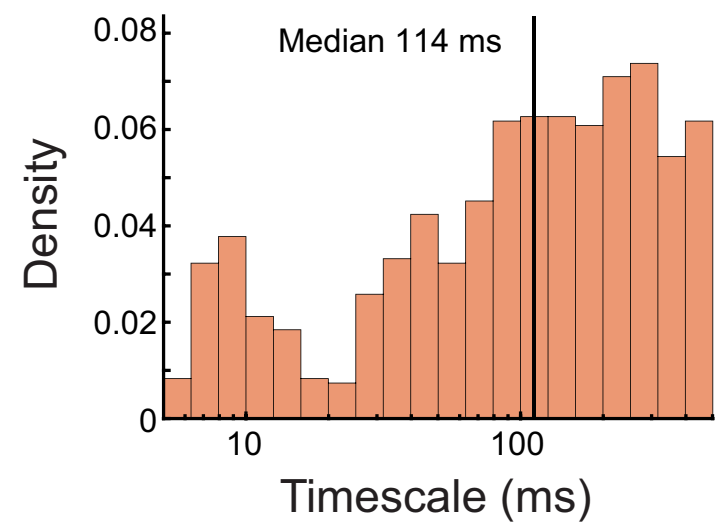

Supplementary Fig. 2 | Distribution of the timescales extracted from the DMS RNNs with rewired synaptic connections. a-c, Rewiring $I \rightarrow I(n=824$; a), $I \rightarrow E(n=1243$; b), or $E \rightarrow I$ $(n=1015 ; \mathbf{c})$ connections shortened the neuronal timescales. d, Shuffling $E \rightarrow E(n=891)$ connections did not alter the distribution significantly. Solid vertical lines represent median $\log (\tau)$. 

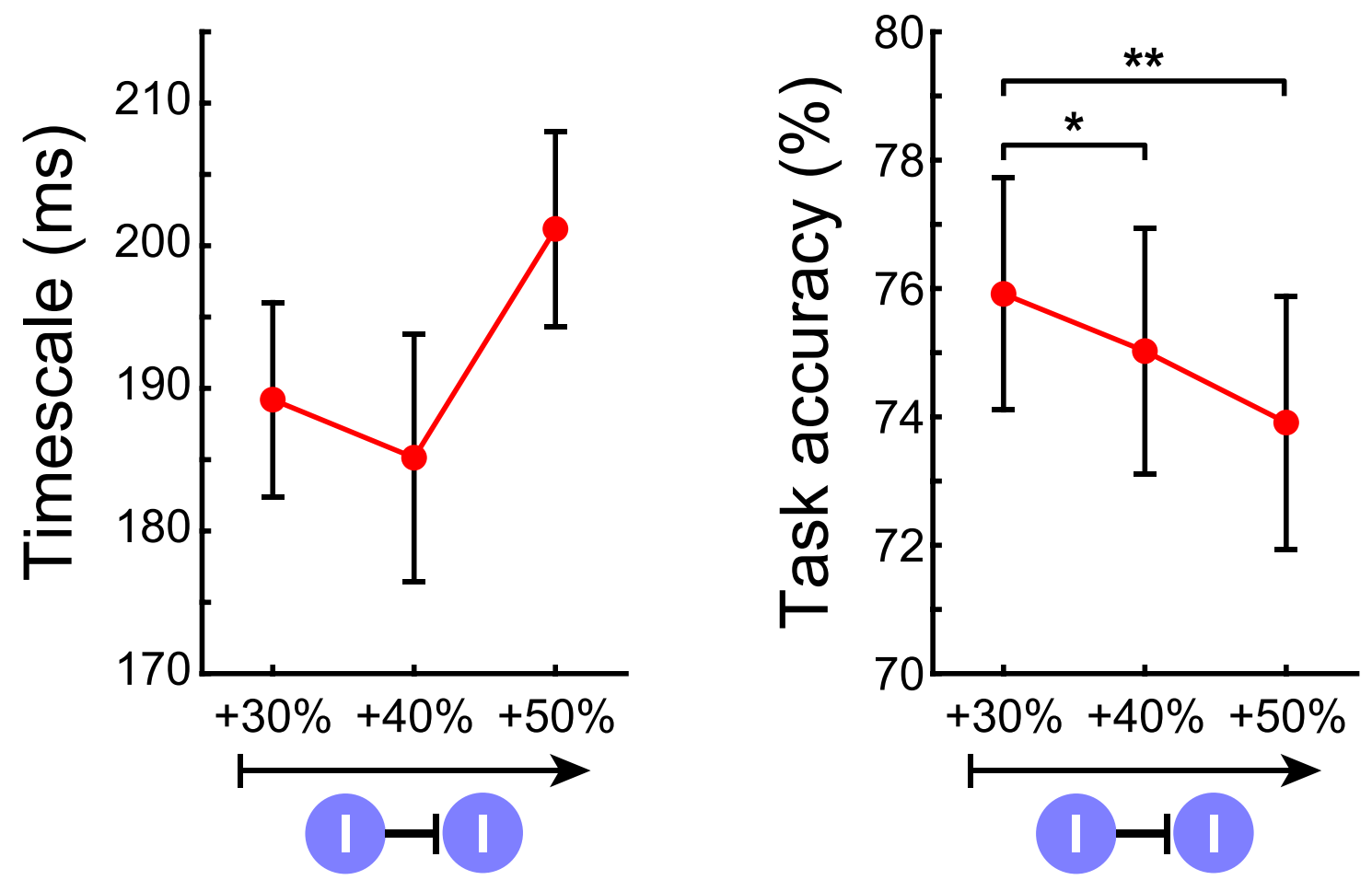

Supplementary Fig. 3 Increasing $I \rightarrow I$ connections does not always lead to increased timescales and task performance. Strengthening $I \rightarrow I$ connections by more than $30 \%$ did not result in significant changes in neuronal timescales (left), but significantly impaired task performance (right). Error bars, \pm s.e.m. ${ }^{*} P<0.05,{ }^{* *} P<0.005$ by two-sided Wilcoxon rank-sum test. 

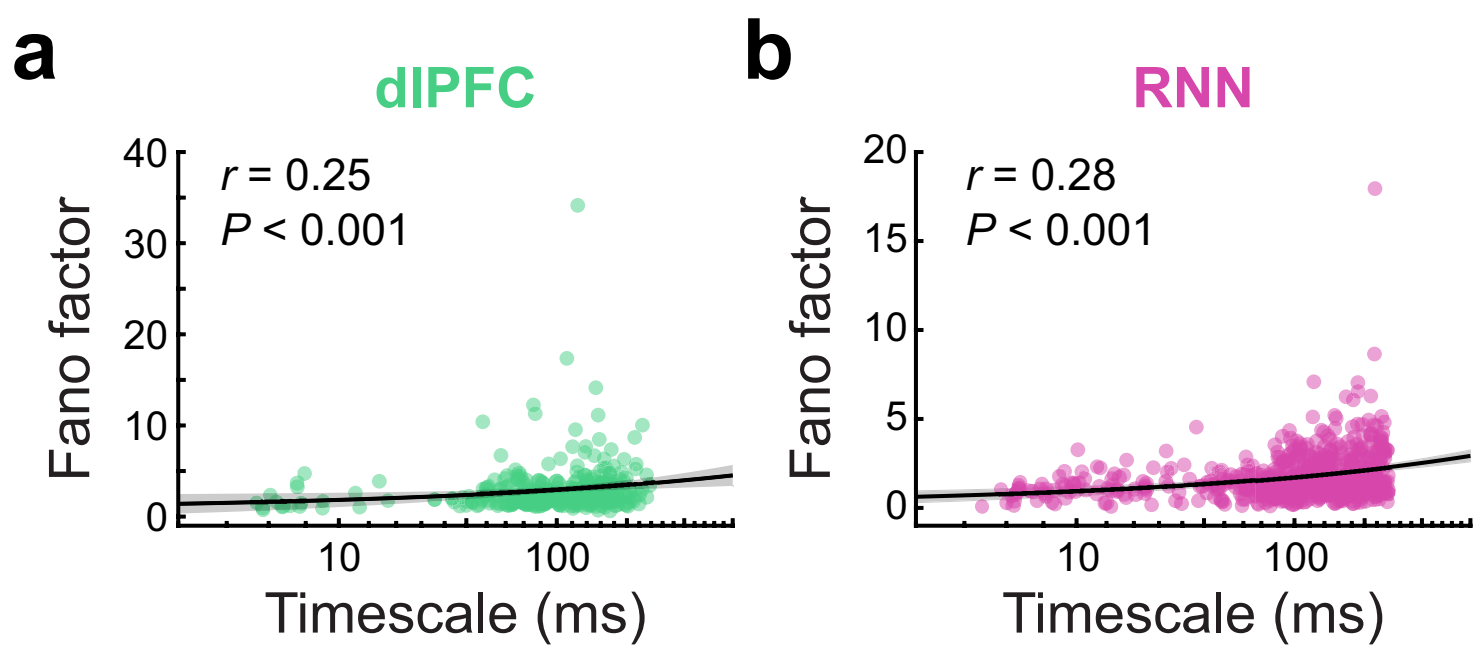

Supplementary Fig. 4 | Relationship between trial-to-trial Fano factors and neuronal timescales. a,b, For both neural (a) and RNN data (b), trial-to-trial spike-count Fano factors were strongly correlated with neuronal timescales. Spearman rank coefficients are shown. Each dot represents a cell or unit. Black solid lines, linear fits to the $\log$-transformed $\tau$; gray shading, $95 \%$ confidence interval of the linear fits. 

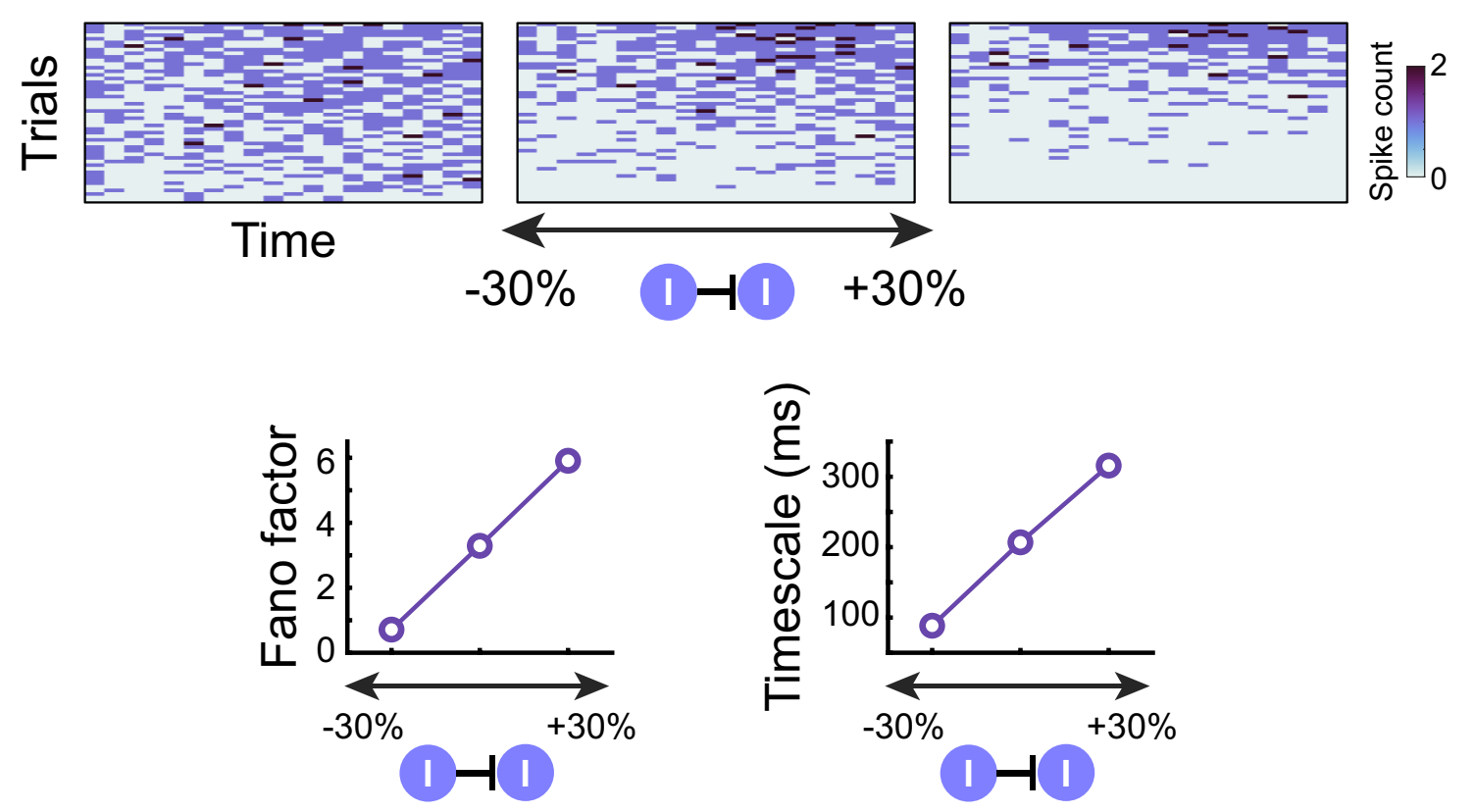

Supplementary Fig. 5 | Example unit whose spontaneous firing activity and timescale were strongly modulated by $\boldsymbol{I} \rightarrow \boldsymbol{I}$ strength. An inhibitory unit from an example DMS RNN displayed highly dynamic baseline firing activity as $I \rightarrow I$ strength increased (top). The unit's Fano factor and timescale increased linearly with increasing $I \rightarrow I$ strength (bottom). 

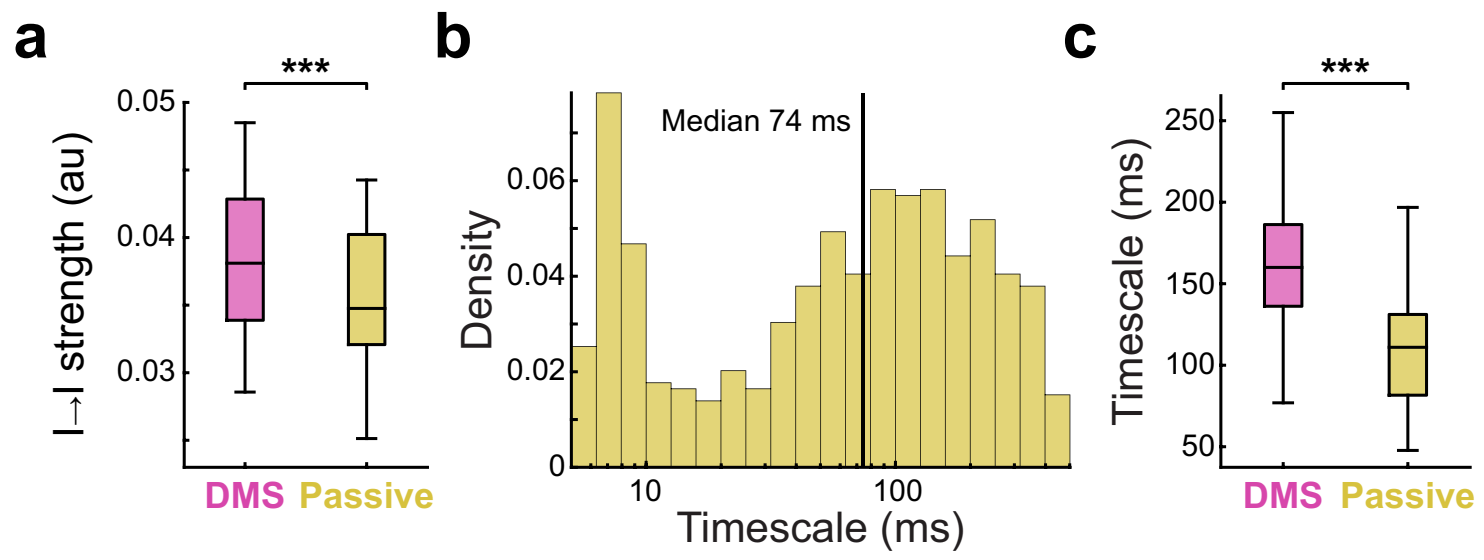

Supplementary Fig. 6 | Training DMS RNNs to perform the passive DMS task by re-tuning recurrent connections $(\boldsymbol{W})$. a, Re-tuning the recurrent connections resulted in weakened $I \rightarrow I$ connection strength. $\mathbf{b}$, Distribution of the timescales $(n=598)$ from the re-trained DMS RNNs. Solid vertical line represents median $\log (\tau)$. c, In line with the decreased $I \rightarrow I$ strength, the timescales from the re-trained RNNs were significantly shorter than those extracted from the DMS RNNs. Boxplot central lines, median; red circles, mean; bottom and top edges, lower and upper quartiles; whiskers, $1.5 *$ interquartile range; outliers not plotted. ${ }^{* * *} P<0.0001$ by Wilcoxon signed-rank test. 